\title{
PERCEPCIÓN Y DISTANCIA ACÚSTICA: LA VARIACIÓN PARAMÉTRICA INDIVIDUAL EN LA ESCISIÓN FONEMÁTICA DE /@/ EN EL ESPAÑOL ANDALUZ. DATOS DE LA CIUDAD DE MÁLAGA
}

\author{
PERCEPTION AND ACOUSTIC DISTANCE: THE INDIVIDUAL PARAMETRIC \\ VARIATION IN PHONEMIC SPLIT OF /@/ IN ANDALUSIAN SPANISH. \\ DATA OF THE CITY OF MALAGA
}

\author{
Álvaro Molina García \\ Universidad de Málaga \\ alvaromolina@uma.es
}

Enviado: 24/09/2018

Aceptado: 09/06/2019

\begin{abstract}
Resumen
En este trabajo se propone un modelo cuantitativo para medir la distancia acústica de las realizaciones de $/ \mathrm{s} / \mathrm{y} / \theta /$ en relación con las grafías $<\mathrm{s}>\mathrm{y}<\mathrm{z}$, ce, ci $>$. Con carácter previo a la construcción de dicho modelo, se demuestra a partir de las realizaciones de doce informantes que este proceso de escisión puede considerarse como una excepción al principio de Garde - tal y como fue entendido por Labov (1994: 485)-; se caracterizan acústicamente los alófonos, y se representa un modelo gráfico basado en el concepto de coordenadas polares, con el fin de reflejar la variación paramétrica individual en la articulación de los alófonos escindidos de $/ \theta /$. Los resultados muestran que los informantes jóvenes instruidos establecen una distancia superior a la de los informantes no instruidos de
\end{abstract} Abstract
In this work a valid model is proposed
to objectively and numerically measure the
acoustic distance of the realizations of $/ \mathrm{s} /$ and
$/ \theta /$ in relation to the letters $<\mathrm{s}>$ and $<\mathrm{z}$, ce,
$\mathrm{ci}>$. Prior to the construction of this model, it
is demonstrated from the realizations of twelve
informants that this process of splitting can be
considered as an exception to Garde's principle
- as it was considered by Labov (1994: 485 )
-; the allophones are acoustically characteri-
zed, and a graphic model based on the concept
of polar coordinates is represented, in order
to reflect the individual parametric variation
in the articulation of the excised allophones
of $/ \theta /$. The results show that educated young
informants establish a distance greater than
that of older uneducated informants and that

1 El presente trabajo se ha realizado en el marco del Proyecto de Investigación Estudio Complementario de los Patrones Lingüísticos del Español de Málaga (ECOPASOS-MA), financiado por el Ministerio de Economía y Competitividad, FFI2015-68171-C5-1 y fondos FEDER

Para citar este artículo / To cite this article: Molina García, Álvaro (2019). Percepción y distancia acústica: la variación paramétrica individual en la escisión fonemática de $/ \theta /$ en el español andaluz. Datos de la ciudad de Málaga. ELUA, 33: 111-140. doi: 10.14198/ELUA2019.33.6

Enlace / Link: http://dx.doi.org/10.14198/ELUA2019.33.6 
mayor edad y que existe una notable variación en los parámetros acústicos subyacentes que cada hablante modifica individualmente.

PALABRAS CLAVE: Variación paramétrica individual, principio de Garde, distancia acústica, escisión fonemática de $/ \mathrm{s} / \mathrm{y} / \theta /$, español de Andalucía. there is a notable variation in the underlying acoustic parameters that each speaker modifies individually.

KEY WORDS: individual parametric variation, Garde's principle, Acoustic distance, phonemic split of /s/ and / $/$ /, Andalusian Spanish.

\section{INTRODUCCIÓN}

\subsection{Marco histórico}

Durante la Edad Media, el sistema de sibilantes del español estaba compuesto por una serie de siete fonemas: africado predorsodental sordo y sonoro /ts/ y /dz/, fricativo apicoalveolar sordo y sonoro /s/ y /z/, fricativo prepalatal sordo y sonoro $/ \mathrm{s} / \mathrm{y} / \mathrm{z} / \mathrm{y}$ africado palatal sordo /t $\mathrm{f} /$ (Alarcos Llorach 1965: 265). Según la opinión más extendida, entre los siglos XV y XVIII ${ }^{1}$, este sistema experimentó un proceso de cambio que tradicionalmente se ha conocido como confusión de sibilantes y que estableció la configuración del panorama fonológico del español peninsular y americano. Como es bien sabido, estos fonemas sufrieron una serie de cambios que determinaron la existencia ulterior de dos soluciones: el sistema del español del centro y norte de la Península y el sistema del español meridional y americano.

Tras los procesos de fricatización de los fonemas africados $(/ \mathrm{ts} />/ \mathrm{s} / ; / \mathrm{dz} />/ \mathrm{z} /)$ y de ensordecimiento de los fricativos $(/ \mathbf{S} /=/ \mathrm{z} / ; / \mathrm{J} /=/ 3 /)$, la oposición de fonemas resultaba un tanto inestable, ya que quedaban tres unidades fricativas sordas cuyo único rasgo de oposición era el punto de articulación y este, a su vez, era muy cercano (Alarcos Llorach 1965: 269-275; Cano Aguilar 2004: 834). Consecuentemente, en el español del centro y norte de la Península se adelantó la articulación del fonema predorsodental ( $/ \mathbf{s} />/ \theta /$ : interdentalización) y se retrasó el prepalatal $(/ \mathrm{J} />/ \mathrm{x} /$ : velarización). De esta manera, a partir del sistema de siete fonemas iniciales, se estableció otro más reducido, compuesto por un fonema africado palatal sordo /t $\mathrm{f} /$ (cacha) y tres fricativos: interdental sordo / $\theta /$ (caza), apicoalveolar sordo /s/ (casa) y velar sordo /x/ (caja). Por razones histórico-sociales - como el hecho de que la Corte se estableciese en Toledo, ciudad del centro de la Península-, esta solución fue considerada como prestigiosa y hoy en día es la adoptada por la variedad estándar del español (Catalán Menéndez-Pidal 1982: 128).

No obstante, en la zona meridional se dio una solución diferente. Los fonemas africados predorsodentales y fricativos apicoalveolares confluyeron en un único fonema dental $/ \theta /$ (Lapesa Melgar 1957: 251; Menéndez Pidal 1962: 104-106; Alarcos Llorach 1965: 276; Catalán Menéndez-Pidal 1982: 123-126; Cano Aguilar 2004: 843-848). La tendencia a realizar alófonos más adelantados o atrasados de este fonema dio como resultado lo que se ha considerado, respectivamente, como ceceo y seseo modernos. En este contexto, el fonema africado palatal experimenta a menudo el mismo proceso de fricatización $(/ \mathrm{t} />/ \mathrm{J} /)$

1 Sin embargo, hay datos que sitúan el origen de este cambio en el siglo XIII (Frago Gracia, 1993: 327; Tuten 2003: 246). 
que afectó a las obstruyentes dentales, ya que no era necesaria su realización africada para oponerse al fonema fricativo y no existía un fonema apicoalveolar /s/ cuyas realizaciones habrían sido muy próximas a los alófonos fricativos (Villena Ponsoda 2001). Por su parte, la realización meridional del fonema velar fue normalmente aspirada ([h]).

Para dar cuenta de la coexistencia de varios patrones de realización de las obstruyentes coronales en ambos sistemas, han surgido tres términos: distinción, que hace referencia a la oposición entre el fonema interdental $/ \theta /$ y el apicoalveolar $/ \mathrm{s} /$, correspondientes, respectivamente, a las grafías $<\mathrm{z}, \mathrm{ce}, \mathrm{ci}>\mathrm{y}<\mathrm{s}>$; seseo, que incluye realizaciones alofónicas sibilantes o estridentes del fonema $/ \theta /$ en las variedades meridionales independientemente de la grafía, y сесеo, que se refiere a las realizaciones alofónicas no sibilantes o mate del mismo fonema.

Málaga ha sido considerada tradicionalmente como una comunidad de habla ceceante (Alvar 1973). Hoy en día, sin embargo, sabemos que en esta ciudad la situación está cambiando y la distinción es actualmente muy frecuente, especialmente entre los jóvenes instruidos (Ávila Muñoz 1994; Cuevas Molina 2001; Lasarte Cervantes 2012; Villena y Vida 2012), de modo que, aparentemente, el proceso de fusión fonemática que se inició en la Edad Media se está revirtiendo parcialmente.

\subsection{Objetivos}

El primer objetivo de este trabajo es comprobar si la fusión de /s/ y / $/$ / que se produjo en la Edad Media llegó a completarse o no, ya que, según el principio de Garde (1961: 38, apud Labov 1994: 485), la fusión total de dos fonemas es irreversible. Indudablemente, solo hay dos opciones aquí: primera, la fusión nunca se completó, sino que estaríamos ante un caso de fusión aparente (Labov 1994: 544-573), de manera que el principio de Garde sería válido; segunda, la fusión fue efectiva, en cuyo caso estaríamos ante una excepción a dicho principio. La hipótesis que sostenemos en este trabajo es que la convergencia llegó a ser total, de modo que son los factores externos los causantes de la escisión contemporánea.

El segundo objetivo consiste en crear un modelo multivariante que refleje cuáles son los parámetros acústicos que resultan significativos a la hora de percibir las realizaciones como [s] o como $[\theta]$, de manera que sea posible alcanzar el tercer y cuarto objetivo.

El tercer objetivo es reflejar la variación paramétrica de la que se valen los individuos para contrastar sus realizaciones en función de la grafía; es decir, demostrar cómo cada individuo basa la distinción entre $/ \mathrm{s} / \mathrm{y} / \theta /$ en parámetros acústicos diferentes en el marco del modelo general.

El cuarto y último objetivo del trabajo, que es el fin de este en sí mismo, consiste en crear un modelo que permita calcular numéricamente en qué grado de progreso o regularización está el proceso de escisión en cada informante, con el fin de disponer de una medida cuantitativa de la distancia articulatoria en función de las grafías $<_{\mathrm{s}}>\mathrm{y}<\mathrm{z}$, ce, ci $>\mathrm{y}$ poder así conocer su distribución social. De esta manera, pretendemos solventar los problemas y dificultades que surgieron en otros métodos postulados con anterioridad (Sawoff 1980; Caravedo 1992; Villena, Ávila y Sáez 1994-1995; Moya y García Wiedemann 1995; Regan 2017). La hipótesis al respecto es que los hablantes jóvenes instruidos presentan una distancia superior a la que muestran los hablantes no instruidos de mayor edad. 


\subsection{Estructura}

El trabajo se organiza de la siguiente manera: en primer lugar, se explica la metodología que se ha seguido: tipo y características del corpus y de la muestra de hablantes, criterios de selección de palabras y esbozo de los tipos de análisis acústicos y estadísticos aplicados (§2). En segundo lugar, se expone brevemente el estado de la cuestión sobre la interpretación fonológica de la fusión fonemática originaria y se presentan los resultados de un análisis acústico con el propósito de comprobar experimentalmente o descartar si existen pistas que permitan argumentar que la escisión se ha producido como efecto de una fusión aparente (§3). En tercer lugar, por último, se caracterizan acústicamente las realizaciones de $[\mathrm{s}]$ y $[\theta](\S 4)$ y se presenta un modelo para cuantificar la distinción (§5). El trabajo se cierra con un breve apartado de conclusiones $(\S 6)$.

\section{METODOLOGÍA}

En este estudio se han analizado las realizaciones de doce informantes procedentes del Corpus oral complementario del español hablado en la ciudad de Málaga (Vida Castro, en prensa). Se trata de una colección de textos orales obtenida en 2013 siguiendo la metodología propia del Proyecto para el Estudio Sociolingüístico del Español de España y América (PRESEEA) con la finalidad central de realizar análisis acústicos de los datos orales. La muestra está compuesta por 54 hablantes, clasificados por género (hombre, mujer), edad (20-34, 35-54 y <55 años) y nivel educacional (bajo, medio y superior). Los informantes seleccionados para este trabajo son doce hombres clasificados por edad (20-34, 35-54 y <55 años) y nivel educacional (sin estudios y con estudios superiores), dos por casilla.

De acuerdo con el principio de accountability (Labov 1972: 72), la selección de palabras no siguió un criterio perceptivo (subjetivo). A partir de la transcripción de la entrevista y con carácter previo a la reproducción, se escogieron las palabras por la grafía para que fuera la posible agrupación de ciertos parámetros acústicos la que determinara si se mantenían separadas las series léxicas con $<\mathrm{z}$, ce, ci $>$ o con $<_{\mathrm{s}}>$, y no la percepción de sonidos. En cuanto al número y características de las palabras, se tomaron en cada entrevista las 20 primeras palabras de cada grafía en posición intervocálica, con un total de 480 realizaciones.

Estas palabras se recortaron y se analizaron con el programa Praat (Boersma y Weenink 2017). Para medir los parámetros que se explican a continuación, se delimitaron las fricativas con ayuda de un TextGrid. Para ello, se seleccionó el ruido de las fricativas como marca de inicio y de final. Puesto que los contextos eran intervocálicos, los formantes vocálicos del espectrograma facilitaron la labor de segmentación.

Para realizar el análisis, se utilizaron tres scripts. El primero, Saveall, es de José Joaquín Atria, del Laboratorio de Fonética Letras de UC- Chile y permite guardar todos los objetos seleccionados de manera que se pueda pasar el siguiente script, el de Wendy Elvira-García, del Laboratori de Fonètica de la Universitat de Barcelona. Este último permite la creación y el almacenado de TextGrid para cada archivo. Finalmente, también se utilizó uno de Elvira-García (Zero-crossings and spectral moments). Este script aplica el filtro Pass Hann band que elimina los sonidos por debajo de $1000 \mathrm{~Hz}$ y por encima de $11000 \mathrm{~Hz}$ y extrae los siguientes parámetros: 
(1) Intensidad $[\mathbf{d B}]$. Tradicionalmente, la intensidad ha sido uno de los parámetros que han resultado más relevantes en la distinción de fricativas (Martínez Celdrán y Fernández Planas 2007: 107; Cicres Bosch 2011: 35; Univaso et alii 2014: 113; Lasarte Cervantes 2012: 144; Regan 2017: 119). Los autores citados registran una intensidad mayor en [s] que en [ $\theta]$. Para este trabajo, el script de Elvira-García permite analizar la intensidad media, la intensidad mínima y la intensidad máxima. La intensidad no se ha estandarizado, dado que los trabajos anteriores han ofrecido resultados similares tanto con la intensidad absoluta como con la estandarizada (Regan 2017: 119).

(2) Parámetros espectrales [Hz]. Se han utilizado cinco. El centro de gravedad (CoG) es una medida que indica la altura media de la frecuencia en la que se produce el ruido de la fricción (Vida Castro 2016: 24). La desviación estándar del espectro «mide la distancia de las frecuencias del espectro respecto del centro de gravedad» (Cicres Bosch 2011: 37). La skewness (asimetría) mide la frecuencia en la que predomina una mayor energía a un lado y a otro del centro de gravedad, de manera que los valores positivos implican que la energía se da principalmente en frecuencias bajas, y viceversa (Cicres Bosch 2011: 40). La curtosis mide el apuntamiento del espectro, de manera que los valores positivos implican espectros puntiagudos, y viceversa (Jongman et alii 2000: 1253). El pico espectral señala en qué frecuencia se produce la mayor intensidad de sonido; es decir, a qué altura se encuentra la mayor concentración de energía (Vida Castro 2016: 24).

Por norma general, se han registrado valores elevados del CoG, skewness y curtosis para [s] y elevados de pico espectral y desviación estándar para [ $\theta$ ] (Norlin 1983: 115; Svantesson 1986: 65; Forrest et alii 1988: 122; Flipsen et alii 1999: 670-671; Jongman et alii 2000: 1256; Gordon et alii 2002: 23; Fox y Nissen 2005: 757; Haley et alii 2010: 5-6; Lasarte Cervantes 2012: 152; Cicres Bosch 2011: 42; Regan 2017: 119). Entre estos parámetros, destaca el $C o G$. Aparentemente, un $C o G$ elevado es indicativo de una sibilancia elevada; de hecho, en el análisis acústico de la denominada [s] ultra sibilada del español del sur de Chile (Sadowsky y Perdomo 2018), se registra un CoG anormalmente elevado $(10630 \mathrm{~Hz})$.

(3) Duración total del segmento [ms]. A pesar de ser un factor que no siempre resulta significativo y que suele tener menor fuerza, la duración también ha sido un factor tradicionalmente utilizado (Jongman et alii 2000: 1255; Gordon et alii 2002: 28; Fox y Nissen 2005: 757; Lasarte Cervantes 2012: 142; Regan 2017: 119). La tendencia es que la $[\mathrm{s}]$ presente una duración mayor que la $[\theta]$.

(4) Cruces por cero. Indica el número de veces que, en un intervalo de tiempo dado, un punto de la onda cruza por el punto cero del eje horizontal (Kiss 2013: 12). Sin embargo, es evidente que la duración del segmento influye en este parámetro. Para solventar este problema, Martínez Celdrán (2015: 123) propone estandarizar la medida multiplicando por 10 el número de cruces por cero y dividiendo el resultado por la duración total del segmento. Este es el valor normalizado que se ofrece en los resultados de este trabajo. A pesar de que no es una medida que se haya utilizado previamente para diferenciar /s/ y $/ \theta /$, resultó ser significativa en la diferenciación de estos sonidos por parte de algunos de los informantes que participaron en el experimento.

Una vez recogidos todos los parámetros en una hoja de cálculo, se ejecutaron diversos análisis estadísticos. Para el primer objetivo — comprobar si existen pistas articulatorias que justifiquen que el proceso de escisión se basa en un caso de fusión aparente- 
se llevó a cabo con el programa Statistical Package for the Social Sciences para Mac, versión 21, el test U de Mann-Whitney, que permite ver si las diferencias de medias de los diversos parámetros acústicos son significativas o no en función de la grafía (§3.2.1). A continuación, tras un test de fiabilidad perceptiva, se ejecutó el mismo análisis, esta vez en función de las realizaciones percibidas, y no de la grafía (§3.2.2). Para realizar la caracterización acústica de [s] y [ $\theta$ ] - el segundo objetivo — se realizó con $R$ Wizard, una interfaz de R desarrollada en la Universidad de Vigo (Guisande et alii 2014), un análisis bivariante (\$4.1.1) cuyo objetivo era servir de orientación para la creación de los modelos multivariantes, obtenidos respectivamente a partir de un análisis discriminante (§4.1.2.1) y otro de regresión logística binomial (§4.1.2.2), del cual se puede extraer una fórmula de predicción de la realización individual de $/ \mathrm{s} / \mathrm{y} / \theta /$. Para el tercer objetivo — visualizar la variación paramétrica individual-, se representó también con RWizard un gráfico de coordenadas polares (\$4.2.2), a partir del cual pudo además medirse la distancia euclídea de las realizaciones ( $\$ 4.2 .3)$, de manera que se consigue el objetivo final del trabajo; esto es, hallar un método para cuantificar de forma objetiva la distancia articulatoria de las realizaciones en correspondencia con la grafía.

\section{FUNDAMENTOS ACÚSTICOS DEL CAMBIO FONÉTICO}

\subsection{Estado de la cuestión}

Como se ha visto en la introducción (§1.2), a pesar de que Garde (1961: 38, apud Labov 1994: 485) defiende que la fusión completa de dos fonemas no puede revertirse, en Málaga y en otras ciudades de Andalucía se está produciendo un caso que parece a priori contrario a este principio; es decir, se está restituyendo la escisión de los fonemas $/ \mathrm{s} / \mathrm{y} / \theta / \mathrm{que}$ se habían fusionado en la Edad Media.

\subsubsection{Fusión aparente}

La primera hipótesis partiría de la idea de que el principio de Garde es válido y universal; es decir, la fusión fonemática completa no puede recuperarse, ya que revertir esta fusión implicaría recuperar los motivos de un cambio lingüístico, el cual es arbitrario (Labov 1994: 486). Dicha hipótesis no consistiría en negar la escisión actual, sino la fusión anterior. Es la teoría del near merger (Labov 1994: 544-573), que puede traducirse como fusión aparente o semifusión. Esta teoría parte de la negación de una premisa tan sencilla y lógica que no se había cuestionado anteriormente: si un hablante pronuncia sonidos de manera diferente, será capaz de discernirlos perceptivamente. Por el contrario, Labov sostiene que en algunos casos es posible cuestionar este aserto. En pares mínimos aparentemente indiferenciables (god-guard), un judío de clase media baja de Nueva York mantiene ciertas diferencias fonéticas en la pronunciación en función de la grafía. Sin embargo, el mismo hablante nativo no es capaz de distinguir perceptivamente los pares que había pronunciado. Esta es la teoría que podría sostenerse para la escisión de las fricativas en Málaga: la fusión nunca se llegó a completar del todo, sino que se produjo una fusión aparente, indiferenciable perceptivamente, pero sí rastreable mediante el análisis acústico de la pronunciación. De ser así, ciertos parámetros acústicos deberían variar en la pronunciación en función de la etimología. 


\subsubsection{Fusión completa}

La segunda hipótesis supone proponer una explicación contraria. El principio de Garde no es válido. Es decir, es posible que dos fonemas que ya se han fusionado completamente puedan escindirse. De hecho, el propio Labov afirma que el principio de Garde no es algo absoluto, sino que - aunque no sea la evolución normal_-, dadas unas determinadas condiciones sociales, puede volver a introducirse una distinción en una comunidad de habla de manera consistente (Labov 1994: 531-532). Hasta tal punto duda Labov de que la reversión sea imposible, que propone algunos factores internos con alta probabilidad de violar el principio de Garde, como la predictibilidad léxica alta y la oposición léxica baja (1994: 514).

Precisamente, la hipótesis que se plantea en este trabajo es que, en realidad, el proceso de escisión fonemática de /s/ y / $\theta$ / sería una de las consecuencias de una corriente de convergencia hacia la variedad estándar nacional, relacionada con los cambios político-sociales surgidos a partir de la Guerra Civil (mediados del siglo XX) en Andalucía ${ }^{2}$, capaz de producir una variedad relativamente intermedia entre el español andaluz y el español centroseptentrional de España.

En la ciudad de Málaga, han estado en contacto desde hace años tres variedades: el estándar nacional, el estándar de prestigio regional (Sevilla) y el vernacular urbano malagueño (Lasarte Cervantes 2012: 130). Sin embargo, la ciudad se encuentra cada vez más alejada de la norma de Sevilla. Esta influencia de la norma urbana sevillana, cada vez más débil en el este de la comunidad, pero aún con cierto vigor en la parte occidental, es una variedad que los hablantes urbanos de las clases socioculturales medioaltas de la ciudad de Málaga tienden a soslayar para aproximarse a la variedad estándar del centro de la península (Villena Ponsoda y Vida Castro 2012). De esta manera, ha surgido una variedad intermedia, que acerca por norma general las variedades usadas por los hablantes jóvenes, urbanos y de nivel sociocultural medioalto de Andalucía a las utilizadas por hablantes de clase trabajadora, rurales y de nivel sociocultural bajo del centro de la península, dando paso a un modelo de pirámide invertida, cuya idea básica es la de prescindir de las características lingüísticas más marcadas y con menor prestigio y mantener aquellas menos prominentes, además de adquirir algunos rasgos del estándar nacional, como es el caso de la distinción entre /s/ y /0/ (Villena Ponsoda y Vida Castro 2017).

\subsection{Análisis acústico}

Con el fin de determinar si existen pistas acústicas de las realizaciones que las separen en función del origen etimológico de la palabra que las representan en la escritura, de tal modo que se pudiese afirmar que la fusión no llegó a completarse, se llevó a cabo un análisis no paramétrico (U de Mann-Whitney) de las diferencias de medias de los parámetros acústicos considerados (vid. §2.2) en las series léxicas con $<_{\mathrm{S}}>$ o con $<\mathrm{z}$, ce, ci $>$ (§3.2.1). A continuación (\$3.2.2), se repitió el análisis considerando, sin embargo, la percepción de las realizaciones.

2 Posiblemente, la Guerra Civil supuso el origen de una serie de cambios sociales a causa de la inestable situación política y los movimientos migratorios, lo cual creó situaciones de grandes diferencias que se reflejaron lingüísticamente, de entre otras maneras, con la formación de una variedad intermedia de convergencia hacia el estándar nacional como consecuencia de la apertura social (Moya y Sosinki 2015: 44-45). 


\subsubsection{Análisis acústico en función de la grafía}

Para la realización del análisis no paramétrico de diferencia de medias de los parámetros acústicos, se hicieron dos análisis separados que permitiesen establecer una comparación en función del nivel de estudios. Como es sabido (vid. §3.1.2), en la ciudad de Málaga son los hablantes instruidos quienes favorecen el proceso de escisión fonemática de $/ \mathrm{s} / \mathrm{y} / \theta / \mathrm{y}$, por tanto, son los hablantes sin estudios los que mantienen la realización interdental con independencia de la grafía. Se esperaba que los hablantes con estudios universitarios polarizasen sus realizaciones en función de la grafía y que los hablantes sin estudios no diferenciasen los parámetros acústicos. Efectivamente, la Tabla 1 muestra cómo los seis hombres del nivel superior de estudios presentan diferencias significativas en todos los parámetros acústicos excepto en la duración, el CoG y la curtosis.

$$
\mathbf{n}=\mathbf{1 2}
$$

\begin{tabular}{|c|c|c|c|c|c|c|c|}
\hline \multirow[b]{2}{*}{ Parámetros } & & \\
\hline & Grafía & Media & Desv. Típica & Sig. & Media & Desv. Típica & Sig. \\
\hline \multirow{2}{*}{ Duración } & $<\mathrm{s}>$ & 94 & 26 & \multirow{2}{*}{.158} & 79 & 18 & \multirow{2}{*}{.936} \\
\hline & $<\mathrm{z}, \mathrm{ce}, \mathrm{ci}>$ & 100 & 30 & & 80 & 23 & \\
\hline \multirow{2}{*}{$\begin{array}{l}\text { Cruces por } \\
\text { cero }\end{array}$} & $<\mathrm{s}>$ & 81 & 30 & \multirow{2}{*}{.006} & 70 & 53 & \multirow{2}{*}{.865} \\
\hline & $<\mathrm{z}, \mathrm{ce}, \mathrm{ci}>$ & 100 & 47 & & 69 & 50 & \\
\hline \multirow{2}{*}{ Int. Mínima } & $<\mathrm{s}>$ & 38 & 5 & \multirow{2}{*}{.000} & 41 & 5 & \multirow{2}{*}{.625} \\
\hline & $<\mathrm{z}, \mathrm{ce}, \mathrm{ci}>$ & 35 & 5 & & 40 & 5 & \\
\hline \multirow{2}{*}{ Int. Máxima } & $<\mathrm{s}>$ & 48 & 5 & \multirow{2}{*}{.001} & 52 & 5 & \multirow{2}{*}{.650} \\
\hline & $<\mathrm{z}, \mathrm{ce}, \mathrm{ci}>$ & 46 & 5 & & 51 & 5 & \\
\hline \multirow{2}{*}{ Int. Media } & $<\mathrm{s}>$ & 45 & 5 & \multirow{2}{*}{.000} & 47 & 5 & \multirow{2}{*}{.718} \\
\hline & $<\mathrm{z}, \mathrm{ce}, \mathrm{ci}>$ & 42 & 5 & & 47 & 5 & \\
\hline \multirow{2}{*}{$\mathrm{CoG}$} & $<\mathrm{s}>$ & 2388 & 1538 & \multirow{2}{*}{.146} & 1537 & 2131 & \multirow{2}{*}{.902} \\
\hline & $<\mathrm{z}, \mathrm{ce}, \mathrm{ci}>$ & 2484 & 2397 & & 1453 & 1958 & \\
\hline \multirow{2}{*}{ Desv. Est. } & $<\mathrm{s}>$ & 2734 & 939 & \multirow{2}{*}{.001} & 2410 & 1932 & \multirow{2}{*}{.907} \\
\hline & $<\mathrm{z}, \mathrm{ce}, \mathrm{ci}>$ & 3410 & 1583 & & 2347 & 1717 & \\
\hline \multirow{2}{*}{ Skewness } & $<$ s $>$ & 2,7 & 2 & \multirow{2}{*}{.020} & 7 & 5 & \multirow{2}{*}{.744} \\
\hline & $<\mathrm{z}, \mathrm{ce}, \mathrm{ci}>$ & 3,6 & 3 & & 7 & 5 & \\
\hline \multirow{2}{*}{ Curtosis } & $<$ s $>$ & 17 & 46 & \multirow{2}{*}{.306} & 98 & 136 & \multirow{2}{*}{.832} \\
\hline & $<\mathrm{z}, \mathrm{ce}, \mathrm{ci}>$ & 28 & 50 & & 88 & 124 & \\
\hline \multirow{2}{*}{ Pico Esp. } & $<\mathrm{s}>$ & 4446 & 1700 & \multirow{2}{*}{.000} & 4913 & 2437 & \multirow{2}{*}{.001} \\
\hline & $<\mathrm{z}, \mathrm{ce}, \mathrm{ci}>$ & 6862 & 2920 & & 5801 & 2759 & \\
\hline
\end{tabular}

\section{Informantes con \\ estudios básicos}

Informantes con

estudios superiores

Para las diferencias de medias, se ha duplicado el test U de Mann-Whitney. En negrita se marcan las diferencias significativas

Tabla 1. Efecto de la etimología/grafía en los correlatos acústicos de /s/ y / $/$ /. Comparación entre hablantes universitarios y hablantes con educación básica 
Un ejemplo visual de cómo estos informantes modifican los parámetros en función de la grafía es el Gráfico 1, que compara dos diagramas de dispersión en los que el eje $y$ mide el pico espectral, y el eje $x$, la medida estandarizada de cruces por cero de un informante de estudios superiores y de un informante de estudios básicos. El gráfico se ha realizado a partir de las realizaciones de un solo informante en cada caso porque, como se verá $(\S 4)$, cada hablante modifica parámetros diferentes para marcar la distinción, por lo que, si se representase el comportamiento de todos los individuos del grupo en un solo gráfico, se superpondrían los parámetros individuales y las diferencias no se verían claras. De hecho, en el gráfico del informante de estudios superiores, a pesar de que se aprecia cómo sus realizaciones se distribuyen en dos grupos en correspondencia con las grafías, el número de cruces por cero de algunas de las realizaciones correspondientes a la grafía $<\mathrm{z}$, ce, ci $>$ es igual o inferior a otras correspondientes a $<\mathrm{s}>$. Aunque los cruces por cero resultan estadísticamente significativos en el conjunto de los datos (Tabla 1), es el pico espectral el parámetro del que verdaderamente se vale este informante para distinguir las realizaciones. Por estas razones, resulta conveniente y necesario construir un modelo en el que entren todos los parámetros que permita reflejar la variación paramétrica individual (vid. §4.2).

Gráfico 1. Diagrama de dispersión individual de las realizaciones de /s/ y / $\theta /$ en el espacio definido por el pico espectral y los cruces por cero. Comparación de los datos de un hablante universitario y de un hablante con estudios básicos

Estudios superiores

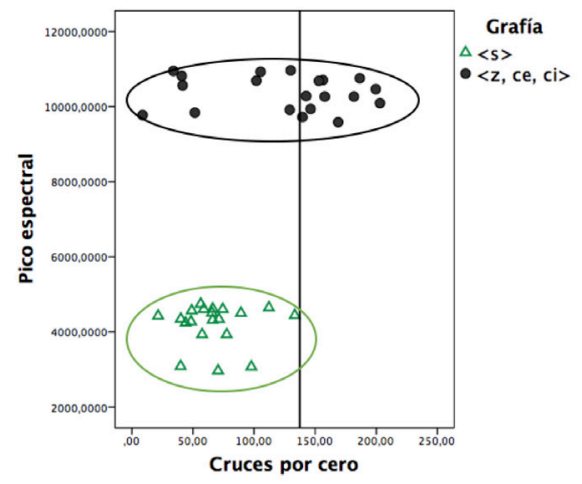

Picoespectral, $<\mathrm{S}>,=4200 ;<\mathrm{z}, \mathrm{ce}, \mathrm{ci}>,=10300$.

Sig. $\mathrm{p}<.001$

Cruces por cero, $<\mathrm{s}>,=67 ;<\mathrm{z}, \mathrm{ce}, \mathrm{ci}>,=124$.

Sig. $\mathrm{p}<.003$

\section{Estudios básicos}

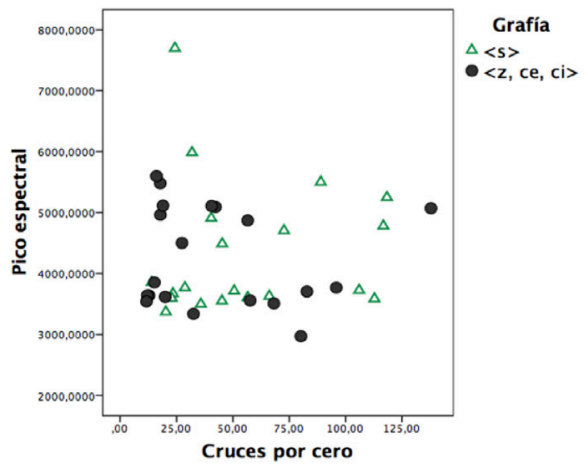

En el caso de los hablantes no instruidos, el panorama es totalmente diferente. La Tabla 1 muestra cómo los parámetros tienen unas medias bastante cercanas en las dos series léxicas (con $<\mathrm{s}>\mathrm{y}$ con $<\mathrm{z}$, ce, ci $>$ ). Los seis hombres con nivel de estudios básicos no modifican significativamente los parámetros acústicos en función de la grafía, a excepción 
del pico espectral. Esto también lo refleja la comparativa del Gráfico 1. En este último caso, las realizaciones alofónicas del informante de estudios básicos varían de manera independiente a la grafía.

Ahora bien, los informantes no instruidos sí diferencian el pico espectral en función de la grafía $(<\mathrm{s}>,=4913 ;<\mathrm{z}, \mathrm{ce}, \mathrm{ci}>,=5801$, vid. Tabla 1$)$, lo cual puede parecer sorprendente. Sin embargo, es posible explicar este hecho: de los seis informantes con estudios básicos, cuatro no modifican ni un solo parámetro en función de la grafía; es decir, solo hay dos informantes que modifican algún parámetro. Son los dos informantes jóvenes, que modifican precisamente el pico espectral: uno $<\mathrm{s}>,=4200$; $<\mathrm{z}$, ce, ci $>,=6600$, y otro $<\mathrm{s}>$, $=5400 ;<\mathrm{z}, \mathrm{ce}, \mathrm{ci}>,=7200$. Son estas medias de los hablantes jóvenes las que alteran la media del grupo, pues los otros informantes no presentan diferencias significativas en el pico espectral. De aquí podría deducirse (Regan 2017: 240) que ciertos informantes justifican la fusión aparente. Sin embargo, hay otra explicación posible: estos hablantes presentan estas medias porque algunas de las palabras que se representan con grafía $<_{\mathrm{S}}>$ en la escritura se perciben como sibilantes. No sería entonces un ejemplo de fusión aparente, ya que - no puede olvidarse - , para que una fusión aparente se considere como tal, debe: a) percibirse igual, y b) articularse diferente (Labov, 1994: 544-573); en el caso de estos dos informantes jóvenes, la primera condición no se cumple. Se trataría de hablantes que diferencian algún parámetro acústico en algunas palabras para acercarse a la pronunciación canónica, básicamente porque son jóvenes, aunque sus estudios sean básicos.

\subsubsection{Análisis acústico en función de las realizaciones percibidas}

En este sentido, se consideró que los ejemplos en los que verdaderamente se prueba si existe fusión aparente son aquellos que se perciben igual (como fricativa interdental sorda $[\theta]$, que es la pronunciación tradicional vernacular de las clases trabajadoras y rurales de Málaga). Se llevó a cabo, pues, el mismo análisis, pero teniendo en cuenta solo aquellas realizaciones que se percibían como fricativa dental sorda [ $\theta]$ (es decir, casos de $<z$, ce, ci $>$ y de $<_{\mathrm{S}}>$ percibidos como $[\theta]$ ), de manera que se comprobase si, a pesar de ser percibidas estas como idénticas, aún habría diferencias significativas en los parámetros.

Con el objeto de comprobar la fiabilidad de la percepción de los alófonos, con carácter previo a este análisis, se realizó un test de fiabilidad perceptiva tendente a corroborar que la percepción era segura ${ }^{3}$. En primer lugar, se etiquetaron las realizaciones en cuatro categorías perceptivas:

3 Con este test también se pretendía comprobar si la duración del alófono tenía relación con que este fuese percibido como $[\mathrm{s}] /[\theta]$, o como realizaciones intermedias o difícilmente categorizables. La hipótesis planteada al respecto es que cuando el hablante tiene la certeza de que el alófono que va a articular se corresponde con la pronunciación canónica según el modelo de la variedad estándar, alarga la articulación. Por otro lado, aquellos hablantes cuyas realizaciones varían en el continuo con independencia de la ortografía articulan alófonos de menor duración. Consecuentemente, se esperaba que las realizaciones percibidas próximas a los polos del continuo de realización de la obstruyente fricativa $([\mathrm{s}]$ y $[\theta])$ durasen más que aquellas difícilmente categorizables y, por tanto, que las realizaciones de los informantes instruidos también durasen más que las de los informantes no instruidos. Los resultados fueron los esperados: la duración es menor en las realizaciones percibidas como intermedias, y mayor en las realizaciones percibidas con claridad: [s]: $96 \mathrm{~ms}$; $\left[\theta^{\mathrm{s}}\right]: 83 \mathrm{~ms}$; $[\theta]: 92 \mathrm{~ms}$; sig. p. $<.000$. Asimismo, como se esperaba, los informantes de estudios superiores presentan una mayor duración en sus realizaciones ( 97 $\mathrm{ms})$ que los informantes de estudios básicos $(80 \mathrm{~ms})$, de manera que se corrobora la hipótesis planteada. 

$[\mathrm{s}]$
2) $\left[\theta^{\mathrm{s}}\right]$
3) $[\theta]$
4) $[\mathrm{h}],[\varnothing]$

Para comprobar si los parámetros acústicos implicados en las realizaciones alofónicas consideradas se diferenciaban significativamente en los diferentes grupos, se realizó el test de diferencia de medias en muestras independientes Kruskal-Wallis. Como se aprecia en la Tabla 2, todos los parámetros utilizados previamente resultaron significativos $\mathrm{p}$ $<.000$. Esto quiere decir que las categorización de las realizaciones que se han percibido auditivamente como $[\theta]$, frente a las percibidas como $[\mathrm{s}]$ o como $\left[\theta^{\mathrm{s}}\right]$, tiene un respaldo objetivo, puesto que todos los parámetros acústicos muestran valores estadísticamente significativos.

Puede así afirmarse que la categorización perceptiva es fiable. Sobre esta base, se analizó si los informantes de estudios básicos modificaban los parámetros acústicos en las realizaciones percibidas como $[\theta]$ en función de la grafía; es decir, se esperaba determinar si las realizaciones $[\theta]$ etimológica (grafía $<\mathrm{z}, \mathrm{ce}, \mathrm{ci}>$ ) y $[\theta]$ no etimológica (grafía $<_{\mathrm{S}}>$ ) de estos hablantes de nivel educacional básicos se diferenciaban de algún modo. De esta manera, se comprobaría si la fusión es total o no. Tal y como se esperaba, no existe ningún parámetro significativo que contraste ambas series (Tabla 3). Las medias de todos los parámetros son prácticamente las mismas. A diferencia del análisis realizado simplemente en función de la grafía (Tabla 1), ahora el pico espectral no resulta significativo. Se prueba así que la diferencia que se vio en el primer análisis en los dos hablantes jóvenes no es consecuencia de una fusión aparente, sino de una articulación y percepción diferentes.

Con el fin de descartar, finalmente, la idea de que haya personas con capacidad de discernir cuándo una realización de $[\theta]$ procede de la grafía $<_{\mathrm{S}}>\mathrm{y}$ cuándo de $<\mathrm{z}$, ce, ci $>$ — pues podría ocurrir que existiesen parámetros que posibilitasen virtualmente la percepción separada pero no hubiesen sido tenidos en cuenta en este trabajo - se realizó un test de percepción para comprobar la discriminación correcta de las series léxicas con $<_{\mathrm{s}}>\mathrm{y}$ con $<\mathrm{z}$, ce, ci $>$. Para el mencionado test, se seleccionaron dos informantes distintos para hacer la grabación: un hombre de estudios básicos de tercera generación y un hombre de estudios universitarios de primera generación. Los jueces fueron 54 personas, clasificadas en tres grupos de edad $(<35,35-55,>55$ años), género y nivel de estudios (básico, intermedio y avanzado), con tres encuestados por casilla (Molina-García 2019). Los resultados de este test de percepción se corresponden con los comentados arriba (vid. Tabla 3): el porcentaje de acierto para las realizaciones procedentes del hablante no instruido con mayoría de realizaciones percibidas como $[\theta]$ es solo del $5,80 \%$, sin variación significativa en función de las características sociolingüísticas de los jueces, en tanto que las realizaciones del informante con estudios universitarios obtuvieron un $97,35 \%$ de acierto. 
Tabla 2. Correlatos acústicos de las realizaciones percibidas como $[\mathrm{s}],\left[\theta^{\mathrm{s}}\right]$ y $[\theta]$ de todos los informantes

\begin{tabular}{|c|c|c|c|c|}
\hline Parámetro & Percepción & Media & Desv. Típica & Sig. \\
\hline \multirow{3}{*}{ Duración } & [s] & 96 & 25 & \multirow{3}{*}{.001} \\
\hline & {$\left[\theta^{s}\right]$} & 83 & 25 & \\
\hline & {$[\theta]$} & 92 & 26 & \\
\hline \multirow{3}{*}{ Cruces por cero } & {$[\mathrm{s}]$} & 86 & 30 & \multirow{3}{*}{.000} \\
\hline & {$\left[\theta^{\mathrm{s}}\right]$} & 71 & 47 & \\
\hline & {$[\theta]$} & 91 & 52 & \\
\hline \multirow{3}{*}{ Intensidad mínima } & [s] & 39 & 5 & \multirow{3}{*}{.000} \\
\hline & {$\left[\theta^{\mathrm{s}}\right]$} & 39 & 5 & \\
\hline & {$[\theta]$} & 37 & 5 & \\
\hline \multirow{3}{*}{ Intensidad Máxima } & {$[\mathrm{s}]$} & 50 & 4 & \multirow{3}{*}{.021} \\
\hline & {$\left[\theta^{\mathrm{s}}\right]$} & 49 & 6 & \\
\hline & {$[\theta]$} & 48 & 6 & \\
\hline \multirow{3}{*}{ Intensidad Media } & {$[\mathrm{s}]$} & 46 & 4 & \multirow{3}{*}{.001} \\
\hline & {$\left[\theta^{s}\right]$} & 45 & 6 & \\
\hline & {$[\theta]$} & 43 & 5 & \\
\hline \multirow{3}{*}{ Centro de gravedad } & {$[\mathrm{s}]$} & 2523 & 1637 & \multirow{3}{*}{.000} \\
\hline & {$\left[\theta^{\mathrm{s}}\right]$} & 1600 & 1976 & \\
\hline & {$[\theta]$} & 2102 & 2373 & \\
\hline \multirow{3}{*}{ Desviación estándar } & [s] & 2896 & 1065 & \multirow{3}{*}{.000} \\
\hline & {$\left[\theta^{\mathrm{s}}\right]$} & 2444 & 1659 & \\
\hline & {$[\theta]$} & 3120 & 1803 & \\
\hline \multirow{3}{*}{ Skewness } & [s] & 2 & 2 & \multirow{3}{*}{.000} \\
\hline & {$\left[\theta^{\mathrm{s}}\right]$} & 6 & 4 & \\
\hline & {$[\theta]$} & 5 & 4 & \\
\hline \multirow{3}{*}{ Curtosis } & [s] & 16 & 34 & \multirow{3}{*}{.000} \\
\hline & {$\left[\theta^{\mathrm{s}}\right]$} & 72 & 113 & \\
\hline & {$[\theta]$} & 48 & 88 & \\
\hline \multirow{3}{*}{ Pico espectral } & [s] & 4857 & 2098 & \multirow{3}{*}{.001} \\
\hline & {$\left[\theta^{\mathrm{s}}\right]$} & 5060 & 2558 & \\
\hline & {$[\theta]$} & 6468 & 3184 & \\
\hline
\end{tabular}

En negrita se marcan las diferencias significativas en el test Kruskal-Wallis 
Tabla 3. Correlatos acústicos en función de la grafía de las realizaciones percibidas como $[\theta]$ de los hablantes de instrucción básica

\begin{tabular}{|c|c|c|c|c|}
\hline Parámetro & Grafía & Media & Desv. Típica & Sig. \\
\hline \multirow{2}{*}{ Duración } & $<s>$ & 86 & 18 & \multirow{2}{*}{.484} \\
\hline & $<\mathrm{z}, \mathrm{ce}, \mathrm{ci}>$ & 85 & 23 & \\
\hline \multirow{2}{*}{ Cruces por cero } & $<s>$ & 90 & 53 & \multirow{2}{*}{.775} \\
\hline & $<\mathrm{z}, \mathrm{ce}, \mathrm{ci}>$ & 88 & 50 & \\
\hline \multirow{2}{*}{ Intensidad mínima } & $<s>$ & 38 & 5 & \multirow{2}{*}{.875} \\
\hline & $<\mathrm{z}, \mathrm{ce}, \mathrm{ci}>$ & 39 & 5 & \\
\hline \multirow{2}{*}{ Intensidad Máxima } & $<\mathrm{s}>$ & 50 & 5 & \multirow{2}{*}{.843} \\
\hline & $<\mathrm{z}, \mathrm{ce}, \mathrm{ci}>$ & 50 & 5 & \\
\hline \multirow{2}{*}{ Intensidad Media } & $<s>$ & 45 & 5 & \multirow{2}{*}{.721} \\
\hline & $<\mathrm{z}, \mathrm{ce}, \mathrm{ci}>$ & 45 & 5 & \\
\hline \multirow{2}{*}{ Centro de gravedad } & $<s>$ & 1846 & 2131 & \multirow{2}{*}{.913} \\
\hline & $<\mathrm{z}, \mathrm{ce}, \mathrm{ci}>$ & 1875 & 1958 & \\
\hline \multirow{2}{*}{ Desviación estándar } & $<s>$ & 3122 & 939 & \multirow{2}{*}{.812} \\
\hline & $<\mathrm{z}, \mathrm{ce}, \mathrm{ci}>$ & 2940 & 1583 & \\
\hline \multirow{2}{*}{ Skewness } & $<s>$ & 5 & 2 & \multirow{2}{*}{.951} \\
\hline & $<\mathrm{z}, \mathrm{ce}, \mathrm{ci}>$ & 5 & 3 & \\
\hline \multirow{2}{*}{ Curtosis } & $<s>$ & 54 & 46 & \multirow{2}{*}{.865} \\
\hline & $<\mathrm{z}, \mathrm{ce}, \mathrm{ci}>$ & 68 & 50 & \\
\hline \multirow{2}{*}{ Pico espectral } & $<\mathrm{s}>$ & 5736 & 1799 & \multirow{2}{*}{.124} \\
\hline & $<\mathrm{z}, \mathrm{ce}, \mathrm{ci}>$ & 6308 & 2920 & \\
\hline
\end{tabular}

Resultados procedentes del test no paramétrico de diferencia de medias U de Mann-Whitney 


\section{ESCISIÓN FONEMÁTICA. FUNDAMENTOS ACÚSTICO-PERCEPTIVOS DE

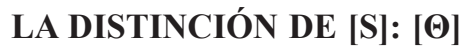

Probado que el caso de la escisión fonemática de [ $\theta]$ en la ciudad de Málaga no se apoya en un caso de fusión aparente, sino que se trata de una escisión — a partir de una fusión que sí llegó a completarse - motivada por un proceso de convergencia hacia la variedad estándar del español (i.e., nuestro el primer objetivo), se muestra a continuación la caracterización acústica de los alófonos $[\mathrm{s}]$ y $[\theta]$ (nuestro segundo objetivo).

\subsection{Bases acústicas de la distinción $[s]:[\theta]$}

\subsubsection{Correlatos acústicos de las obstruyentes fricativas dentales. Modelo bivariante}

Para la caracterización de las obstruyentes fricativas en este estudio, se han calculado las medias no en función de la grafía, sino de aquellas realizaciones percibidas como [s] y $[\theta]$; es decir, los casos en los que la percepción no ofreció duda alguna (vid. §3.2.2). En primer lugar, se realizó un análisis bivariante para cada parámetro acústico (Tabla 4) con el objetivo de orientar el análisis multivariante. Los resultados obtenidos aquí de las obstruyentes fricativas coinciden en general con los de estudios anteriores. Como se aprecia en la Tabla 4, [s] tiende a realizarse más intensa, con el CoG más elevado y más larga que [ $\theta]$, en tanto que tiene valores más bajos de cruces por cero, skewness, curtosis, desviación estándar y pico espectral ${ }^{4}$. La única diferencia que se aprecia en nuestro caso es que, en las realizaciones analizadas, se invierten los valores de skewness y curtosis; es decir, son más altos en $[\theta]$ que en $[\mathrm{s}]$.

\subsubsection{Efecto paramétrico conjunto sobre la percepción. Modelos multivariantes}

Una vez realizado el análisis bivariable, en el que se comprobó que la [s] y la [ $\theta$ ] percibidas son acústicamente diferentes, y dado que son varios los parámetros influyentes, conviene saber ahora qué jerarquía de peso o efecto existe sobre la realización percibida cuando consideramos un modelo multivariable que incluye como variables predictoras a todos estos parámetros.

4.1.2.1. Jerarquía de efectos paramétricos sobre la percepción diferenciada de [s] y $[\theta]$. Análisis discriminante

El análisis discriminante permite caracterizar elementos a partir de un conjunto de variables independientes (funciones discriminantes); es decir, su objetivo es encontrar las variables que separan dos grupos; en este caso, $[\mathrm{s}]$ y $[\theta]^{5}$. De esta manera, disponemos de un modelo que predice qué rasgos permiten percibir - y con qué fuerza - una realización u otra; esto es, discriminar qué serie de variables condicionan la percepción de la obstruyente como sibilante [s] o como no sibilante [ $\theta]$. El análisis se realizó con $R$ Wizard, una interfaz

4 La duración y la desviación estándar, a pesar de no resultar significativas estadísticamente, siguen la tendencia de lo registrado en estudios anteriores: valores más elevados en [s].

5 Sobre el procedimiento de cálculo de variables y coeficientes, vid. Guisande González et alii (2011: 787-810). 
de R desarrollada en la Universidad de Vigo (Guisande González et alii 2014), que permite ejecutar análisis complejos de forma intuitiva.

Tabla 4. Correlatos acústicos de las realizaciones percibidas como $[\mathrm{s}] \mathrm{y}[\theta]$ de todos los informantes

\begin{tabular}{|c|c|c|c|c|}
\hline Parámetro & Realización & Medias & $\begin{array}{l}\text { Desv. } \\
\text { Típica }\end{array}$ & Significación \\
\hline \multirow{2}{*}{ Duración } & {$[\mathrm{s}]$} & 96 & 24 & \multirow{2}{*}{.126} \\
\hline & {$[\theta]$} & 92 & 27 & \\
\hline \multirow{2}{*}{ Cruces por cero } & {$[\mathrm{s}]$} & 86 & 53 & \multirow{2}{*}{.676} \\
\hline & {$[\theta]$} & 91 & 50 & \\
\hline \multirow{2}{*}{ Intensidad mínima } & {$[\mathrm{s}]$} & 39 & 5 & \multirow{2}{*}{.000} \\
\hline & {$[\theta]$} & 37 & 5 & \\
\hline \multirow{2}{*}{ Intensidad Máxima } & [s] & 50 & 4 & \multirow{2}{*}{.008} \\
\hline & {$[\theta]$} & 48 & 6 & \\
\hline \multirow{2}{*}{ Intensidad Media } & {$[\mathrm{s}]$} & 46 & 4 & \multirow{2}{*}{.000} \\
\hline & {$[\theta]$} & 43 & 5 & \\
\hline \multirow{2}{*}{ Centro de gravedad } & {$[\mathrm{s}]$} & 2523 & 1637 & \multirow{2}{*}{.000} \\
\hline & {$[\theta]$} & 2102 & 2373 & \\
\hline \multirow{2}{*}{ Desviación estándar } & {$[\mathrm{s}]$} & 2896 & 1065 & \multirow{2}{*}{.960} \\
\hline & {$[\theta]$} & 3120 & 1803 & \\
\hline \multirow{2}{*}{ Skewness } & {$[\mathrm{s}]$} & 2 & 2 & \multirow{2}{*}{.000} \\
\hline & {$[\theta]$} & 5 & 4 & \\
\hline \multirow{2}{*}{ Curtosis } & [s] & 16 & 34 & \multirow{2}{*}{.000} \\
\hline & {$[\theta]$} & 48 & 88 & \\
\hline \multirow{2}{*}{ Pico espectral } & [s] & 4857 & 2098 & \multirow{2}{*}{.006} \\
\hline & {$[\theta]$} & 6468 & 2909 & \\
\hline
\end{tabular}

En negrita se marcan las diferencias significativas en el test U de Mann-Whitney

El Gráfico 2 es un diagrama de cajas extraído del análisis discriminante, en el cual los lados verticales de las cajas representan el recorrido intercuartílico (la diferencia entre el primer y el tercer cuartil en una distribución) de las puntuaciones canónicas; las cajas se encuentran divididas por un segmento horizontal que indica dónde se posiciona la mediana, y los bigotes marcan los valores extremos de estas puntuaciones. Se aprecia, a la derecha, cómo los valores positivos de intensidad media y centro de gravedad caracterizan a [s] y los valores negativos de skewness, pico, desviación estándar y cruces por cero, a [ $\theta]$.

La Tabla 5 muestra los coeficientes canónicos que subyacen en el Gráfico 2; es decir, la fuerza discriminante que tiene cada variable. Como puede observarse, los parámetros acústicos estadísticamente significativos $(\mathrm{p}<0,05)$ aparecen ordenados en función de la jerarquía de su efecto en la percepción de las obstruyentes fricativas como [s] o [ $\theta$ ]. Dado que el análisis se orientó en el valor de aplicación de [s] percibida, se entiende que aquellos casos con valores altos de intensidad media y centro de gravedad, y con valores bajos de skewness, pico, desviación estándar y cruces por cero, tendrán coeficientes canónicos positivos. 
Gráfico 2. Diagrama de cajas de las realizaciones percibidas como [s] - [ $\theta]$ de todos los informantes extraído del análisis discriminante

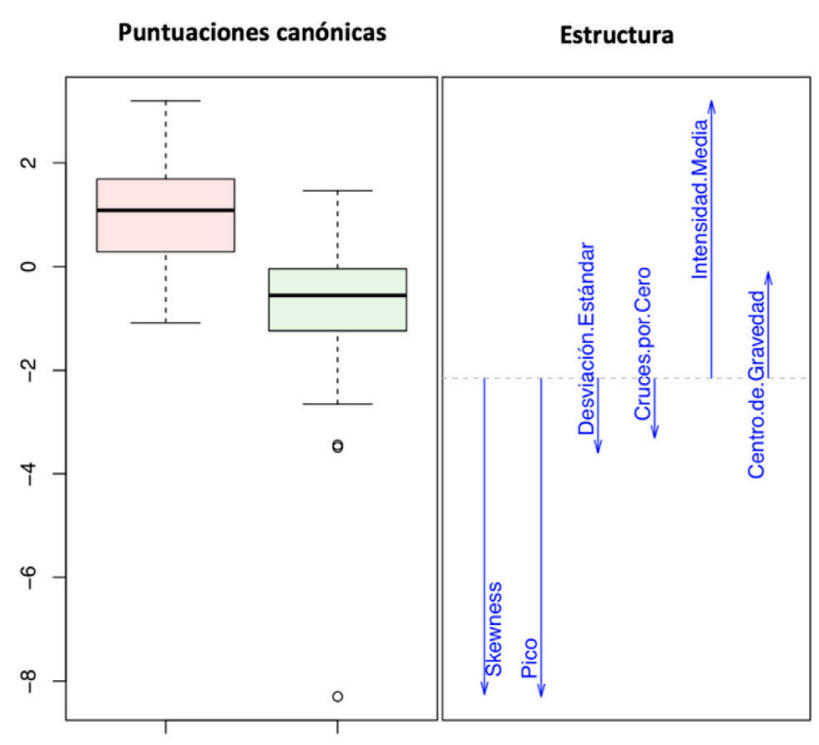

[s]

[0]

Realización percibida

Valor de aplicación: 1 [s]

Tabla 5. Coeficientes canónicos del Análisis Discriminante

Variables

Intensidad Media

Centro de Gravedad

Skewness

Pico

Desviación Estándar

Cruces por Cero

\section{Coeficientes canónicos}

\begin{tabular}{|c|}
\hline 0,40 \\
\hline 0,15 \\
\hline$-0,45$ \\
\hline$-0,45$ \\
\hline$-0,10$ \\
\hline$-0,10$ \\
\hline
\end{tabular}

Valor de aplicación: 1 [s]

Puesto que algunas variables que resultaban claramente significativas en el análisis individual (intensidad mínima, curtosis) no forman parte del modelo, y que otras que no resultaban significativas sí aparecen en él (cruces por cero, desviación estándar), podría suponerse cierta contradicción. Nada más lejos de la realidad. Por una parte, hay que señalar que en un modelo multivariante, de aquellos factores que tienen una elevada correlación positiva entre sí, solo entran aquellos con mayor tolerancia; es decir, las variables independientes que explican un mayor porcentaje de variación que no pueden explicar las demás de la variable dependiente. Esto explica que la intensidad mínima y la intensidad máxima 
no formen parte del modelo. Por otra parte, una distancia amplia entre las medias de una variable no tiene por qué implicar per se una capacidad discriminante elevada. En el caso de la curtosis $([\mathrm{s}],=16, \pm 34 ;[\theta],=48, \pm 88)$, hay una distribución irregular. Posiblemente, algunos casos aislados eleven la media, de manera que esta variable resulta significativa, pero no tiene capacidad predictiva en el modelo, dado que en la mayoría de casos se distribuirá de manera aleatoria o su efecto será pequeño. Por otro lado, variables que presenten poca variación (ni siquiera significativa), pueden tener una fuerza predictiva mayor, de manera que sí se incluyen en el modelo.

El porcentaje de casos identificados correctamente por validación cruzada es elevado tanto en el análisis discriminante lineal, como en el cuadrático ( $79 \%$ y $78 \%$ respectivamente). Este dato es indicativo de un análisis discriminante robusto, ya que la cercanía de ambos porcentajes implica que el análisis es correcto.

4.1.2.2. Predicción de la discriminación perceptiva de $[\mathrm{s}]$ y [ $[\theta]$. Análisis de regresión logística binomial

A continuación, se realizó un análisis de regresión logística binomial por dos razones: la primera es que, en el caso de que el porcentaje de acierto de este modelo fuese similar al del análisis discriminante, se corroboraría la fuerza y replicabilidad de este modelo; la segunda es que este análisis permite calcular la probabilidad de ocurrencia de una variable binomial (en este caso, $0=[\mathrm{s}], 1=[\theta]$ ) en función de una serie de variables independientes (aquí, los parámetros acústicos). Es decir, de este análisis se extrae un modelo cuyo resultado será la probabilidad de que lo que se está articulando sea percibido como [s] o como $[\theta]$ en función del efecto de los parámetros acústicos ${ }^{6}$. Para el cálculo del modelo, se utilizó — como en el análisis discriminante del apartado anterior-RWizard (Guisande González et alii 2014).

En primer lugar, se ejecutó el análisis con la totalidad de los casos. Los resultados refuerzan la solidez del modelo. El porcentaje de identificación es similar al obtenido en el análisis discriminante (83\%). Asimismo, las variables que conforman el modelo son las mismas y apuntan en la misma dirección: valores elevados de intensidad y centro de gravedad para [s], y valores elevados de skewness, pico, desviación estándar y cruces por cero para $[\theta]$ (Tabla 6).

Lo realmente interesante de este tipo de análisis es que, a partir de los coeficientes de la Tabla 6, se genera una fórmula de predicción de la realización. Es decir, se obtiene una ecuación que permite calcular la probabilidad de que una realización sea percibida como [s] o como $[\theta]$. La ecuación resultante de este análisis es:

$\pi=\overline{1+e^{-(-1,10-1,25 * \text { IntMedia }-0,85 * \text { CentroGrav }+4,1 * \text { Skewn }+2,2 * \text { DesvEst }+1 * \text { Cruc }+0,8 * \text { Pico })}}$

6 Sobre el procedimiento de cálculo, vid. Guisande González et alii (2018: 228-236). 
Tabla 6. Coeficientes canónicos del Análisis de Regresión Logística Binomial

\begin{tabular}{|l|c|c|}
\multicolumn{1}{|c|}{ Variables } & Coeficientes canónicos & Significación \\
\hline (Intercept) & $-1,10$ & $\mathbf{. 0 0 0}$ \\
\hline Intensidad Media & $-1,25$ & $\mathbf{. 0 0 0}$ \\
\hline Centro de Gravedad & $-0,85$ & $\mathbf{. 0 5}$ \\
\hline Skewness & 4,10 & $\mathbf{. 0 0 0}$ \\
\hline Desviación Estándar & 2,20 & $\mathbf{. 0 0 0}$ \\
\hline Cruces por Cero & 1 & $\mathbf{. 0 1}$ \\
\hline Pico & 0,80 & $\mathbf{. 0 0 0}$ \\
\hline
\end{tabular}

Valor de aplicación: $1[\theta]$

De esta manera, si un informante, por ejemplo, realiza un alófono con una intensidad media de - 0,75 ; un centro de gravedad de - 0,25 ; un skewness de 0,8 ; una desviación estándar de 0,8; 0,2 cruces por cero, y un pico espectral de -0,5 (parámetros estandarizados para ser comparables entre sí), habría una probabilidad del $97 \%$ de que este alófono fuese percibido como $[\theta]$. De este modo, se consigue un valor numérico extraído del conjunto de variables, que, al fin y al cabo, es lo que percibe el oyente. En este ejemplo, el hecho de que tuviera un pico espectral con valores negativos y un bajo número de cruces por cero, no impediría que fuese percibida como $[\theta]$, ya que las otras variables compensarían.

Es cierto que el porcentaje de casos identificados correctamente es un parámetro fiable para valorar la calidad del modelo, pero aún más fiable resulta comprobar el grado de acierto con datos que no se habían incluido en la matriz original del modelo (Guisande González et alii 2018: 233). Con el objeto de comprobar esto, se crea un modelo a partir de las realizaciones de 11 de los 12 informantes y este modelo se aplica posteriormente al informante excluido para determinar si dicho modelo es capaz de predecir de igual forma cuáles serían sus realizaciones. De esta manera, en el caso de que los datos coincidiesen, se comprobaría la capacidad del modelo para predecir correctamente las realizaciones de otros informantes no consideradas para su construcción y, por tanto, reflejaría que la probabilidad de acierto del modelo es fiable.

Como se esperaba, el porcentaje de acierto del modelo es similar tanto en los 11 informantes como en el informante que no había entrado en la construcción de dicho modelo: $82 \%$ y $83 \%$ respectivamente. A su vez, el porcentaje de acierto también es similar al de los modelos procedentes del análisis discriminante $(79 \%, \S 4.1 .2 .1)$ y del análisis de regresión logística binomial $(83 \%, \S 4.1 .2 .2)$. Se puede concluir que los dos modelos extraídos del análisis multivariable tienen coherencia interna, fiabilidad y replicabilidad.

Un último análisis, por fin, nos sirvió para afianzar la replicabilidad del modelo. Tal análisis consiste en construir el modelo con las mismas variables empleadas anteriormente e ir aplicándolo a cada una de las ocurrencias de [s] y/o [ $\theta]$. En primer lugar, con RWizard se crea una base de datos en la que se desordenan aleatoriamente todas las 
realizaciones. A continuación, se ejecuta el análisis, que consiste en ir aplicando el modelo extraído del análisis de regresión logística previo a cada una de las ocurrencias, de manera que se va acumulando el porcentaje de acierto global. Evidentemente, se espera que este porcentaje vaya subiendo a medida que se introduce un mayor número de casos. Existen dos resultados posibles: a) el porcentaje de aciertos no deja de subir, en cuyo caso se deduciría que el modelo se ajustaría mejor si se introdujesen más realizaciones; b) hay un punto en el que el porcentaje se estabiliza, de lo que se deduciría que, aunque se introdujesen nuevas realizaciones no tenidas en cuenta, el porcentaje de acierto no variaría. Según los análisis realizados hasta el momento, se esperaba que el resultado fuese el segundo. Efectivamente, tal y como refleja el Gráfico 3, a partir de aproximadamente 70 realizaciones el modelo comienza a estabilizarse, y, finalmente, a partir de 160 se asintotiza la función con un porcentaje de acierto medio del 83,65\%; esto es, el mismo que ya se había obtenido. En realidad, este supone una comprobación aún más objetiva y fiable, si cabe, de los análisis de regresión por pasos desarrollados más arriba. De esta manera, se ha logrado el segundo objetivo: determinar cuáles son los parámetros acústicos que utilizan los hablantes para diferenciar las realizaciones de $[\mathrm{s}] \mathrm{y}[\theta]$.

Gráfico 3. Porcentaje de acierto del modelo del análisis de regresión logística por realizaciones escogidas al azar

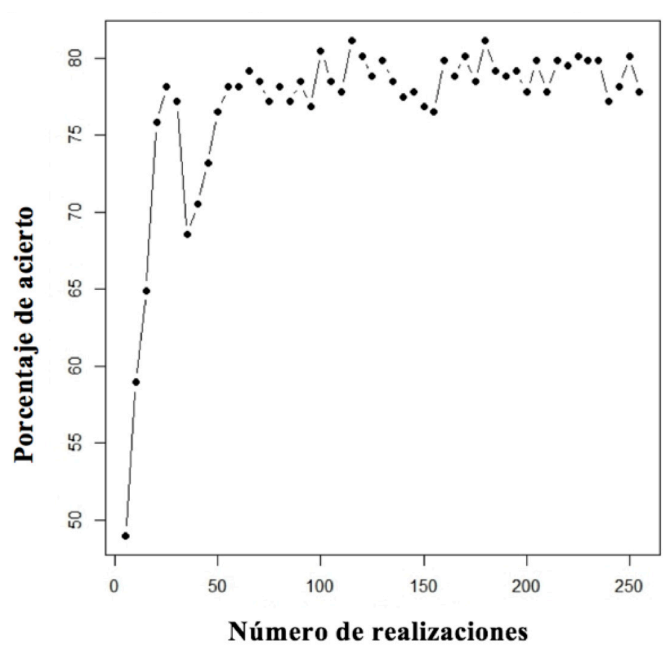

\section{VARIACIÓN PARAMÉTRICA INDIVIDUAL}

\subsection{Antecedentes}

A pesar de que — como se ha comprobado hasta aquí- existe una tendencia general que marca una distinción entre las realizaciones percibidas de los dos fonemas, en el análisis individual de cada informante surgían casos que, en principio, no encajaban dentro del modelo. Estos casos son variados; por ejemplo, uno de los informantes de estudios 
superiores establece una clara distinción perceptiva entre $[\mathrm{s}]$ y $[\theta]$, pero mantiene prácticamente la misma media de intensidad - esto es, el parámetro más influyente en el análisis discriminante (4.1.2.1) - en los dos alófonos.

Cabe recordar que el proceso de escisión fonemática está en curso; se trata, por tanto, de un momento de variación y cambio. Los hablantes tratan de realizar dos alófonos diferentes en función de la pronunciación canónica de la grafía, producto de la escisión que nos ocupa, pero cada uno se vale de su propia selección de parámetros. Todos los informantes cuentan con — valga la metáfora — el mismo piano alofónico, que les permite, en este caso, establecer el contraste. Estaría compuesto por diferentes teclas que cada informante podría pulsar en función de sus circunstancias personales, sus antecedentes, etc., unas teclas, que serían los parámetros analizados; y cada informante tocaría unas teclas. Prueba de ello es que parámetros como la intensidad y los momentos espectrales han sido utilizados en estudios fonéticos forenses de identificación de hablantes, debido a su alta diferencia interpersonal, ya que estos parámetros acústicos varían en función del punto de articulación y de las características físicas y formales de los aparatos fonadores de los hablantes (Cicres Bosch 2011; Univaso et alii, 2014).

Esta variación paramétrica individual se refleja a la perfección cuando se elabora un gráfico bidimensional de dispersión. Según el modelo desarrollado en el apartado 4, la intensidad media y el pico espectral son parámetros con mucha fuerza para diferenciar las realizaciones de $[\mathrm{s}]$ y $[\theta]$. Sin embargo, cuando consideramos el conjunto de los hablantes (Gráfico 4), aparecen muchos casos que no tendrían explicación, puesto que son realizaciones percibidas como $[\mathrm{s}]$ en posiciones esperables para $[\theta]$ y viceversa.

Esto sucede precisamente porque en el análisis individual hay algunos informantes que no utilizan estos mismos parámetros para contrastar $[\mathrm{s}]$ y $[\theta]$ - hecho este esperable, puesto que, en caso contrario, el modelo construido sería fiable al 100\%-. Ahora bien, hay hablantes que, aunque no se basan en los parámetros más significativos, logran articular dos sonidos que son percibidos claramente como distintos. Hay que tener en cuenta que, al final, lo que el oyente percibe es un único sonido caracterizado por todos los parámetros fonéticos. Se trata de una percepción global de un conjunto compuesto por muchos factores. Un ejemplo bastante ilustrativo de esto lo encontramos en el trabajo de Gordon et alii (2002: 161). A pesar de que este, al estudiar el contraste entre las obstruyentes fricativas en siete lenguas, encuentra de forma significativa un centro de gravedad más elevado en [s] que en [f], constata que hay informantes individuales que realizan valores de [s] muy cercanos o incluso superiores en [f].

Sobre esta base, si bien es necesaria la consideración individual de las pautas de realización, tal y como hemos hecho hasta aquí, la correlación bilateral entre los parámetros fonéticos y los alófonos percibidos como fundamento del cálculo de dichas pautas resulta insuficiente. De este modo, gráficos de dispersión similares a los que hemos mostrado (Gráfico 4; vid. asimismo Regan 2017: 256), en los que subyacen correlaciones bilaterales, deberían sustituirse por cálculos y representaciones gráficas que reflejen la idea de la selección individual de parámetros para marcar el contraste efectivo $-\mathrm{o}$ su inexistencia — entre alófonos. Esta variación paramétrica individual recoge más fielmente la realidad de la actuación lingüística de los hablantes y sugiere la imagen de la modulación individual (habla) de los límites colectivos. El instrumento y la técnica multivariable denominada gráfico de coordenadas polares sirve perfectamente a este propósito. 
Gráfico 4. Diagrama de dispersión de las realizaciones percibidas como [s] y [ $\theta$ ] de todos los informantes en el espacio bidimensional definido por el pico espectral y la intensidad media

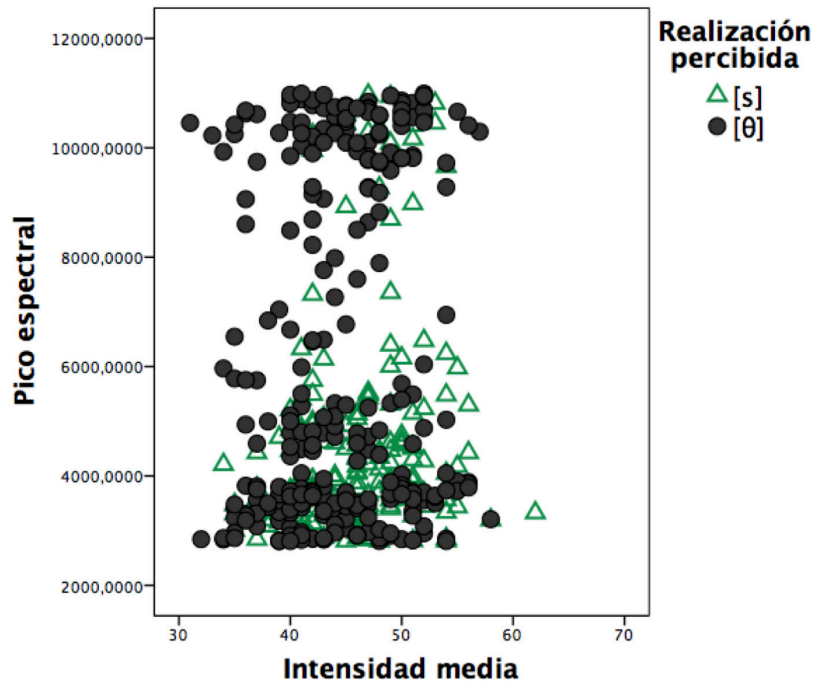

5.2. Representación multidimensional de la variación. Gráfico de coordenadas polares

Un gráfico de coordenadas polares permite visualizar la influencia de un amplio número de variables independientes sobre la variable dependiente. De este modo, frente a un gráfico de dispersión en el que solo se visualiza la posición de la variable dependiente en el espacio definido por la correlación entre dos parámetros, en el gráfico de coordenadas polares se muestra la combinación exacta de los ocho parámetros acústicos subyacentes en la articulación, por parte de cada hablante, de los alófonos percibidos ${ }^{7}$. Con carácter previo a la construcción del modelo y, por tanto, a la elaboración del mencionado gráfico, las medidas de los parámetros deben estandarizarse para que sean comparables entre sí. Las medidas estandarizadas se han calculado automáticamente con el programa SPSS, con la función guardar valores tipificados como variables, en la que subyace la fórmula básica de estandarización:

$$
\mathrm{Z}_{1}=\frac{\mathrm{X}_{1}-\overline{\mathrm{X}}}{\sigma}
$$

Una vez estandarizados los datos, se calculó la media de los valores de los ocho parámetros acústicos correspondientes a las realizaciones de cada palabra en cada uno de los

7 Se han tenido en cuenta todos los parámetros del análisis excepto intensidad mínima y máxima por su mutua correlación positiva con la intensidad media, puesto que su inclusión habría sesgado el gráfico. Es decir, el informante que estableciese alguna diferencia en la intensidad reflejaría una distancia mayor de la real por el hecho de haber medido la intensidad de tres maneras distintas. 
informantes y se agruparon en función de la grafía, de manera que apareciesen representados en el espacio bidimensional del gráfico de coordenadas polares 24 puntos. De esta manera, disponemos ahora de la posición de las realizaciones promediadas de cada hablante relacionadas con la grafía y podemos observar, medida en un espacio multidimensional definido acústicamente, la distancia entre ellas. A continuación, con RWizard (Guisande González et alii 2014) se obtuvieron las coordenadas polares de estos puntos; es decir, se asignaron ángulos a las variables, de acuerdo con el protocolo definido por Guisande González et alii (2011: 1-19). Obtenidos los valores para cada parámetro, el programa permite realizar el gráfico de coordenadas polares. El orden en el que se introducen las variables es importante. Con el argumento COR = FALSE, las variables se ordenan en función de una correlación positiva, de manera que se visualiza mejor la agrupación de casos y la distancia que se refleja entre las realizaciones (Guisande González et alii 2018: 150). Como se señaló anteriormente, el objetivo de este análisis, más allá de visualizar y medir la distancia de las realizaciones de $[\mathrm{s}]$ y $[\theta]$, es reflejar qué parámetros utiliza cada informante para diferenciar las realizaciones.

El Gráfico 5 tiene una explicación simple. Por un lado, los círculos acompañados del número del hablante en nuestro archivo y de la letra $<\mathrm{z}>$ (por ejemplo, $\cdot 12 \mathrm{z}$ ) representan la media de todos los valores paramétricos de los alófonos en las palabras con grafía $<\mathrm{z}$, ce, ci $>$, y los cuadrados acompañados de la letra $<\mathrm{s}>$ (por ejemplo, 12s) indican la media correspondiente a los valores paramétricos de los alófonos en las palabras con grafía $<\mathrm{s}>$. Los números, por su parte, sirven para clasificar por su edad y nivel educacional a los hablantes de la siguiente manera:

a) 1-6: Estudios Básicos

1-2: Jóvenes $(20-34$ años $)$

3-4: Adultos (35 - 54 años) 5-6: Mayores ( $<55$ años)

b) 7-12: Estudios Superiores

7-8: Jóvenes (20 - 34 años) 9-10: Adultos (35 - 54 años) 11-12: Mayores $(<55$ años $)$

Según lo esperado, el Gráfico 5 refleja cómo los informantes de estudios básicos realizan prácticamente todos los alófonos en la zona para la grafía $<\mathrm{z}, \mathrm{ce}, \mathrm{ci}>$ - lo que tradicionalmente se ha conocido como ceceo-y los informantes de estudios superiores son los únicos que muestran sus realizaciones de [s] fuera del área de la grafía $<\mathrm{z}$, ce, ci $>$. La excepción la conforman los informantes 11 y 12 (> 55 años, estudios universitarios), que se encuentran en la frontera. Como se expuso más arriba (\$3.1.2) y se probará a continuación (§5.3), los informantes mayores tienden a seguir utilizando las realizaciones fusionadas y, consecuentemente, a no distinguir los alófonos de [s] y [ $\theta]$. Ahora bien, en los casos comentados, el hecho de que tengan estudios superiores es lo que explica el porcentaje relativamente elevado de correspondencia de la grafía y la realización (en torno al 80\%).

De este modo, se consigue el tercer objetivo: corroborar la hipótesis de que los hablantes contrastan las realizaciones alofónicas basándose en parámetros distintos (variación paramétrica individual). Por un lado, puede apreciarse un primer grupo, formado por los informantes 7, 8, 10 y 11; es decir, aquellos con dos cuadrados unidos por una flecha continua que diferencian las realizaciones basándose fundamentalmente en valores positivos de intensidad y centro de gravedad en $<\mathrm{s}>$, y de skewness y curtosis en $<\mathrm{z}$, ce, ci $>$. No ocupan 
Gráfico 5. Gráfico de coordenadas polares de las realizaciones de las obstruyentes fricativas clasificadas por las grafías $<\mathrm{s}>\mathrm{y}<\mathrm{z}$, ce, ci $>$ de todos los informantes

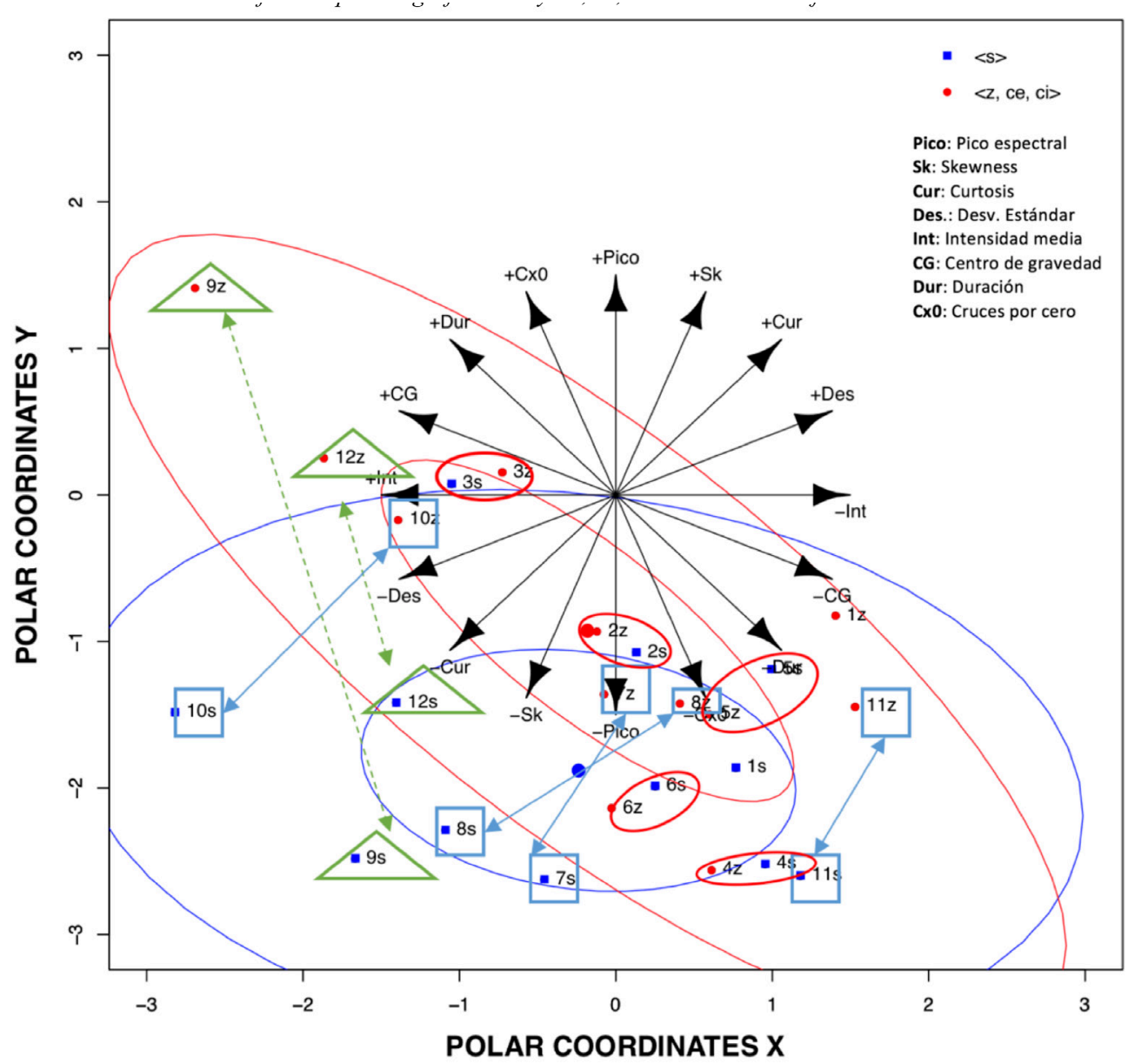

Los círculos concéntricos son a un gráfico de coordenadas polares lo que las cajas y los bigotes a un gráfico de cajas (vid. §4.1.2.1). En el Gráfico 5, se aprecian dos círculos grandes con otros dos círculos de menor tamaño y un punto en su interior. En el círculo horizontal que ocupa la parte inferior del gráfico, el punto central es la media de todas las realizaciones — de todos los informantes - procedentes de palabras con grafía $<_{\mathrm{S}}>$; el círculo interior a este refleja el recorrido intercuartílico, y el círculo grande representa los bigotes. El otro conjunto de círculos con inclinación diagonal representa lo mismo para las realizaciones procedentes de palabras con grafía $<\mathrm{z}, \mathrm{ce}, \mathrm{ci}^{8}$.

8 Evidentemente, puesto que en este gráfico los cálculos se basan en la diferencia en función de la grafía, y no de las realizaciones percibidas, no representa las zonas prototípicas de realización de $[\mathrm{s}] \mathrm{y}[\theta]$ percibidas y, por tanto, la separación de los círculos no es tan marcada como sucede en el gráfico de cajas (4.1.2.1. Gráfico 2). No obstante, si tenemos en cuenta que la mitad de los informantes son instruidos y, consecuentemente, tienden a presentar un patrón distinguidor, nos sirve como guía de la zona que debería ocupar cada realización percibida según el modelo canónico de escisión fonemática 
exactamente la misma zona del gráfico, pero sí se ve cómo contrastan las realizaciones respectivas con una línea recta diagonal ascendente hacia la derecha de unos 50/60 . Por otro lado, los informantes 9 y 12 conformarían otro grupo (aquellos con dos triángulos unidos por una flecha discontinua), que fundamenta la distinción principalmente con valores elevados de pico espectral para $<\mathrm{z}$, ce, ci $>$. La oposición queda marcada en este caso con una línea recta diagonal ascendente hacia la izquierda de unos $100^{\circ}$.

Así pues, en el mencionado gráfico se percibe con claridad cómo los seis informantes de estudios superiores (7-12) establecen una separación espacial entre sus realizaciones mayor que la mostrada por los hablantes de estudios básicos. Las realizaciones correspondientes a la grafía $<s>$ de los informantes $7(x=-0,45 ; y=-2,6), 8(x=-1,1 ; y=-2,3), 9(x=-1,7 ; y=-2,5) y$ $10(x=-2,8 ; y=-1,5)$ se encuentran alejadas de las realizaciones de esos mismos hablantes que corresponden a la grafía $<\mathrm{z}, \mathrm{ce}, \mathrm{ci}>$ : $7(\mathrm{x}=-0,1 ; \mathrm{y}=-1,3), 8(\mathrm{x}=0,4 ; \mathrm{y}=-1,4), 9(\mathrm{x}=-2,7 ; \mathrm{y}=1,4)$ y $10(x=-1,4 ; y=-0,2)$. Por el contrario, las realizaciones correspondientes a las grafías $<\mathrm{s}>$ $\mathrm{y}<\mathrm{z}$, ce, ci $>$ de los informantes de estudios básicos (2-6, excepto el hablante número 1) se encuentran prácticamente en el mismo punto (aparecen agrupadas en círculos en el gráfico), de lo que puede inferirse que la distribución de sus realizaciones respecto a la grafía es aleatoria.

\subsection{Variación y distancia}

La distancia entre la posición de los alófonos de /s/ y / $/$ / en el espacio multidimensional definido por los ocho correlatos acústicos se puede medir numérica y objetivamente. De este modo, se consigue relacionar la distancia entre las realizaciones con la correspondencia gráfica. Se obtiene así: a) un análisis más completo que un simple porcentaje de correspondencia entre grafía y realización percibida, sin tener en cuenta el grado de proximidad entre esas realizaciones y b) una medida más en consonancia con la valoración social de las variantes $\mathrm{y}$, por tanto, más realista. Dada la variación gradual existente en el continuo $[\theta-\mathrm{s}]$, puede ocurrir que un hablante muestre realizaciones cercanas a los dos extremos, $\mathrm{y}$, sin embargo, llevar a cabo dichas realizaciones aleatoriamente — esto es, sin correspondencia sistemática con la ortografía - en tanto que otro hablante podría tener realizaciones cercanas, pero bien polarizadas en función de la grafía. Dicho de otro modo, en este análisis se obtiene un valor numérico que da importancia tanto a la correspondencia de la grafía y la realización, como a la distancia entre los alófonos realizados.

De esta manera, la gran distancia que presenta, por ejemplo, el informante $9\left(<_{\mathrm{S}}>\right.$ : $\mathrm{x}=$ $1,7 ; \mathrm{y}=-2,5 ;<\mathrm{z}$, ce, ci $>$ : $\mathrm{x}=-2,7 ; \mathrm{y}=1,4)$ en este gráfico es el reflejo de un hablante cuyas realizaciones para la grafía $<\mathrm{s}>$ se perciben como [s] y cuyas realizaciones para la grafía $<\mathrm{z}$, ce, ci $>$ se perciben como [ $\theta$ ] en el $100 \%$ de los casos y que, a su vez, diferencia acústicamente con claridad los alófonos [s] y [ $\theta$ ]. Sin embargo, el informante $1(<\mathrm{s}>$ : $\mathrm{x}=0,75, \mathrm{y}=-1,85 ;<\mathrm{z}$, ce, $\mathrm{ci}>\mathrm{x}=1,4, \mathrm{y}=-0,8)$, a pesar de tener una correspondencia de la grafía y la realización del $70 \%$, presenta una distancia inferior debido a que sus alófonos $[\mathrm{s}]$ y $[\theta]$ se encuentran cercanos, lo que explicaría la percepción indiferenciada o errónea del $30 \%$ de los casos.

En el caso de poder medir esta distancia, estaríamos en condiciones de medir de una forma objetiva cómo de avanzado se encuentra el grado de distinción de un informante dado, algo que es relevante aquí y que se ha medido de diversas formas con anterioridad (Sawoff 1980; Caravedo 1992; Villena, Ávila y Sáez 1994-1995; Moya y García Wiedemann 1995; Regan 2017). 
De manera general, los problemas principales que planteaban estos trabajos eran los siguientes: 1) tenían en cuenta la correspondencia entre la realización del hablante y la grafía, pero no en qué medida separaban acústicamente las realizaciones; 2) excluían los casos de realizaciones intermedias, o 3) corrían el riesgo de sobreentender — casi todos ellos- que los casos de [ $\theta$ ] procedente de $<\mathrm{z}$, ce, ci $>$ y [s] procedente de $<\mathrm{s}>$ son realizaciones canónicas o distinguidoras, cuando no tiene por qué ser así (Villena, Ávila, Sáez 1994-1995: 407-408). Frente a ellos, el cálculo de la distancia de realizaciones en función de la grafía a partir del gráfico de coordenadas polares hace posible: 1) reflejar la variación paramétrica individual; es decir, representar en qué parámetros se basa cada informante para distinguir sus realizaciones, 2) comprobar si la variación de las realizaciones de los informantes en el continuo de las obstruyentes fricativas (incluidas las realizaciones intermedias) es aleatoria o se hace de acuerdo a la grafía; esto es, si el proceso de escisión está avanzado, y 3) estudiar el proceso de distinción / indistinción de manera general, sin prestar especial atención a la consideración de cada ocurrencia como un caso de ceceo, seseo o ceseo, y evitar así los problemas de tener que considerar cada realización como seseante, ceceante o canónica, ya que se analizan todas las realizaciones simultáneamente.

Con el fin de medir esta distancia, se aplicó la siguiente fórmula, o cálculo de la distancia euclídea, que no es más que una aplicación práctica del Teorema de Pitágoras, que permite medir la distancia en un sistema de coordenadas cartesianas, donde $\delta$ es la distancia euclídea; y $P_{1}$ y $P_{2}$ son los dos puntos de correspondencia $<\mathrm{s}>$ y $<\mathrm{z}$, ce, ci $>$ en las realizaciones alofónicas de un hablante determinado; $x_{1}$ y $x_{2}$ corresponden a dichos valores en el eje $x$ del gráfico, en tanto que $y_{1}$ e $y_{2}$ se refieren a los valores en el eje $y$ :

$$
\delta\left(P_{1}, P_{2}\right)=\sqrt{\left(x_{2}-x_{1}\right)^{2}+\left(y_{2}-y_{1}\right)^{2}}
$$

Dada la posición relativa de cada conjunto de realizaciones para cada grafía y para cada hablante en el espacio definido por los ocho parámetros acústicos considerados (Gráfico 5), la distancia euclídea entre el punto correspondiente al promedio de realización $<_{\mathrm{s}}>\mathrm{y}$ el punto correspondiente a $<\mathrm{z}$, ce, ci $>$ de un hablante dado nos proporcionará el grado de separación efectivo entre los alófonos de $[\mathrm{s}]$ y $[\theta]$ en función de la grafía.

Establecido así un procedimiento para representar numéricamente la distancia entre el promedio individual de los parámetros acústicos utilizados para realizar las obstruyentes fricativas, es posible analizar sobre una base sólida qué factores sociales determinan esta distancia. Por ejemplo, es muy probable, según la hipótesis planteada y a la vista de la distancia que se aprecia visualmente, que los informantes de estudios superiores presenten distancias significativamente superiores en las realizaciones alofónicas a las de los hablantes de estudios básicos. Para corroborar esta hipótesis, se realizó un análisis de comparación de la diferencia de medias para grupos independientes. Como muestra la Tabla 7, las medias de distancia entre los alófonos de $[\mathrm{s}]$ y $[\theta]$ son significativamente diferentes en los grupos educacionales (intervalo de 1,5) y de edad (intervalo de 0,5). Es decir, los informantes con instrucción superior separan claramente las realizaciones alofónicas en función de la grafía $(=2,0, \pm 0,9)$, en tanto que la distancia es mucho menor entre los hablantes de estudios básicos $(=0,5 \pm 0,3)$. 
Tabla 7. Efecto de las variables de hablante en la distancia acústica de [s] y [ $\theta$ ] en función de la grafía

\begin{tabular}{|c|c|c|c|c|}
\hline Variable & Variante & Distancia & Desv. Típica & Significación \\
\hline \multirow{2}{*}{ Nivel de estudios } & Superiores & 2 & 0,9 & \multirow{2}{*}{.000} \\
\hline & Básicos & 0,5 & 0,3 & \\
\hline \multirow{2}{*}{ Edad } & $1^{\mathrm{a}}-2^{\mathrm{a}}$ Generación & 1,4 & 1,1 & \multirow{2}{*}{.002} \\
\hline & $3^{\mathrm{a}}$ Generación & 0,9 & 0,5 & \\
\hline
\end{tabular}

En negrita se marcan las diferencias significativas en el test U de Mann-Whitney

Como se observa en la Tabla 7 , se recodificó la variable edad en solo dos grupos ( $1^{\mathrm{a}}-2^{\mathrm{a}}$

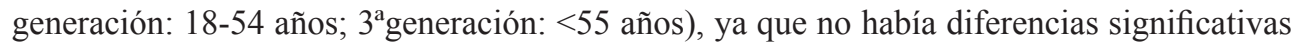
entre las dos primeras generaciones, pero sí entre estas y la tercera. Se puede apreciar cómo los hablantes de más de 55 años son los que tienden a no marcar la distinción fonemática $(=0,9, \pm 0,5)$, lo cual refuerza la hipótesis. Teniendo en cuenta estos resultados y dada la situación de la comunidad de habla (vid. §3.1.2), se esperaba que se diesen cuatro posibilidades en función del grado de avance de la escisión fonemática: $1^{a}$ ) informantes jóvenes de nivel educacional alto, $2^{\mathrm{a}}$ ) informantes mayores de nivel educacional alto, $3^{\mathrm{a}}$ ) informantes jóvenes de nivel educacional básico y $4^{\mathrm{a}}$ ) informantes mayores de nivel educacional básico. Basándonos en la idea de la formación de la variedad intermedia (vid. §3.1.2), se entiende que los valores de la distancia descenderán siguiendo la jerarquía de los grupos establecidos arriba. Esto significa que esperaríamos que los dos polos extremos estuviesen ocupados, respectivamente, por los jóvenes con estudios superiores - con una distancia alofónica media muy elevada en función de la grafía - y por los mayores con estudios básicos — con una distancia mínima - El grupo central estaría formado por los informantes mayores con nivel educacional alto y los jóvenes con nivel educacional básico. La Tabla 8 muestra cómo esta predicción se cumple de manera significativa.

Tabla 8. Variación de la distancia acústica de [s] y [ $\theta]$ en función de cuatro grupos de informantes

Informante

Distancia Desv. Típica Significación

Jóvenes estudios superiores

Mayores estudios superiores

Jóvenes estudios básicos

Mayores estudios básicos

Total

\begin{tabular}{|c|c|c|}
\hline 4 & 2,25 & 1 \\
\hline 4 & 1,4 & 0,2 \\
\hline 4 & 0,55 & 0,4 \\
\hline 4 & 0,4 & 0,1 \\
\hline 12 & 4,6 & 1,7 \\
\hline
\end{tabular}

En negrita se marcan las diferencias significativas en el test Kruskal-Wallis

De esta manera, se consigue poner en relación las variables de hablante con un valor numérico que representa la distancia de las realizaciones en correspondencia con la grafía —el 
cuarto objetivo - y se corrobora la hipótesis planteada al inicio. Por un lado, los hablantes jóvenes instruidos realizan dos alófonos muy separados en el continuo de la obstruyente fricativa $[\mathrm{s}]-[\theta]$, y realizan de forma sistemática el alófono [s] cuando corresponde la grafía $<\mathrm{s}>$ y el alófono [ $\theta$ ] cuando corresponde la grafía $<\mathrm{z}$, ce, ci $>$; esto es, presentan el patrón distinguidor. Por otro lado, los hablantes de mayor edad no instruidos distribuyen aleatoriamente sus realizaciones en función de la grafía; esto es, presentan el patrón reductor. En medio quedan los hablantes mayores con estudios superiores y los jóvenes sin estudios, que tienden a establecer una relación canónica entre los alófonos y la grafía, pero cuyas realizaciones alofónicas no están tan separadas acústicamente, de manera que su percepción en ocasiones no resulta clara.

\section{RECAPITULACIÓN, CONCLUSIONES Y PERSPECTIVAS}

Los objetivos de este trabajo fueron: 1 ) determinar si el proceso de escisión $/ \theta />/ \mathrm{s} /: / \theta /$ es una excepción al principio de Garde (1961: 38, apud Labov 1994: 485) —que establece que un proceso de fusión completa es irreversible - o un proceso basado en un caso de fusión aparente; 2) realizar una caracterización acústica de $[\mathrm{s}]$ y $[\theta]$; 3) representar la variación paramétrica individual en la realización de los alófonos, y 4) medir de manera objetiva la distancia articulatoria de los alófonos en función de la grafía; esto es, hasta qué punto distinguen los hablantes sus realizaciones por la grafía y, por tanto, tienen interiorizado el proceso de escisión fonemática.

A la vista de los resultados, se probó, en primer lugar, que aquellas realizaciones procedentes de hablantes no instruidos percibidas como fricativas interdentales $[\theta]$ no varían significativamente en función de la grafía (3.2). Consecuentemente, puede inferirse que no existen pistas acústicas en la actuación lingüística de estos hablantes — perceptivamente ceceantes y poco proclives a la acomodación en el sentido del contraste fonológico estándar-que hayan permitido revertir la fusión; esto es, no se trata de un caso de fusión aparente, sino que, por el contrario, nos encontramos ante una excepción al principio de Garde motivada por un proceso de convergencia hacia la variedad estándar del español, coherente con la convergencia de otros rasgos lingüísticos en el mismo sentido (Villena Ponsoda y Vida Castro 2012).

En segundo lugar, se registraron en los análisis bivariantes (4.1.1) valores más elevados de intensidad, centro de gravedad y duración para [s], y de cruces por cero, desviación estándar, skewness, curtosis y pico espectral para [ $\theta$ ]. Los análisis multivariantes (4.1.2.1 y 4.1.2.2) ofrecieron resultados similares: valores positivos de intensidad media y centro de gravedad para [s], y de skewness, desviación estándar, cruces por cero y pico espectral para $[\theta]$, con un porcentaje de acierto por validación cruzada del $79 \%$ y el $83 \%$ respectivamente.

En tercer lugar, se constató que existe una gran variación individual a la hora de marcar la distancia acústica de [s] y [ $\theta$ ] en relación a las grafías $<_{\mathrm{s}}>\mathrm{y}<\mathrm{z}$, ce, ci $>$ (5.2). Asimismo, se reflejó cómo los informantes no instruidos no diferencian sus realizaciones en función de la grafía y los informantes instruidos sí lo hacen.

Finalmente, frente a procedimientos previos para cuantificar el progreso relativo del proceso de escisión en la comunidad de habla (Sawoff 1980; Caravedo 1992; Villena, Ávila y Sáez 1994-1995; Moya y García Wiedemann 1995; Regan 2017), en este trabajo se propone un modelo nuevo para calcular el valor numérico de la distancia de los alófonos en función 
de la grafía, de manera que se pueda analizar su relación con las variables sociales (5.3). Los resultados corroboraron la hipótesis: son los hablantes jóvenes instruidos quienes muestran una distancia superior en las realizaciones de los alófonos en función de la grafía; los hablantes de mayor edad no instruidos presentan una distancia inferior, y tanto los jóvenes sin estudios como los mayores con estudios ocupan la posición intermedia.

En próximos estudios, se ampliará la muestra al conjunto de hablantes del Corpus oral complementario del español hablado en la ciudad de Málaga (Vida Castro, en prensa), con 54 informantes, clasificados por edad, género y nivel educacional, de manera que pueda reflejarse de manera representativa la situación actual de la comunidad de habla de la ciudad de Málaga, con especial insistencia en el papel de las variables intermedias y de pequeña escala (Villena Ponsoda y Vida Castro 2012).

\section{REFERENCIAS BIBLIOGRÁFICAS}

Alarcos Llorach, E. (1965). Fonología española, I. Madrid: Gredos.

Alvar López, M. (1973). Notas de asedio al habla de Málaga. Málaga: Ayuntamiento de Málaga.

Ávila Muñoz, A. (1994). "Variación reticular e individual de s/z en el Vernáculo Urbano Malagueño: Datos del barrio de Capuchinos", Analecta Malacitana, 17, pp. 343-367.

Boersma, P. y D. Weenink (2017). Praat: Doing phonetics by computer (versión 6.0.29) [Computer Software]. Amsterdam, Department of Language and Literature, University of Amsterdam.

Cano Aguilar, R. (2004). "Cambios en la fonología del español durante los siglos XVI y XVII". En Cano Aguilar, R. (coord.). Historia de la lengua española. Barcelona: Ariel, pp. 825-857.

Caravedo, R. (1992). “¿Restos de distinción /s/ / / en el español del Perú?”, Revista de Filología Española, 72, pp. 639-654.

Catalán Menéndez-Pidal, D. (1982). "El fin del fonema /z/ en español”. En Marcos Marín, F. (coord.). Introducción plural a la gramática histórica. Madrid: Cincel.

Cicres Bosch, J. (2011). "Los sonidos fricativos sordos y sus implicaciones forenses", Estudios Filológicos, 48, pp. 33-48.

Cuevas Molina, I. (2001): Variación social y reticular de las consonantes obstruyentes dentales $\left(/ \theta^{s} /\right)$ y palatales (/c/) en el Vernáculo Urbano Malagueño (VUM). Tesis Doctoral, Málaga: Universidad.

Flipsen, P. Jr., L. Schriberg, G. Weismer, H. Karlsson y J. Mcsweeny (1999). "Acoustic characteristics of /s/ in adolescents", Journal of Speech, Language and Hearing Research, 42, pp. 663-677.

Forrest, K., L. Shriberg, G. Weismer, H. Karlsson y J. McSweeny (1988). "Statistical analysis of Word-initial voiceless obstruents: Preliminary data", Journal of the acoustic society of America, 84, pp. 115-124.

Frago Gracia, J. A. (1993). Historia de las hablas andaluzas. Madrid: Arco Libros.

Fox, R. y S. Nissen (2005). "Sex-related acoustic changes in voiceless English fricatives", Journal of Speech, Language and Hearing Research, 48, pp. 753-765.

Garde, P (1961). "Réflexions sur les différences phonétiques entre les langues slaves", Word, 17, pp. 34-62.

Gordon, M., P. Barthmaier y K. Sands (2002). "A cross-linguistic acoustic study of voiceless fricatives", Journal of the International Phonetic Association, 32/2, pp. 141-174.

Guisande González, C., A. Vaamonde Liste y A. Barreiro Felpeto (2011). Tratamiento de datos con R, Statistica y SPSS. Madrid: Díaz de Santos.

Guisande González, C., J. Heine, J. González-DaCosta y E. García-Roselló (2014). RWizard Software, Universidad de Vigo.

Guisande González, C., J. Heine, J. González-DaCosta y E. García-Roselló (2018). "RWizard Manual": http://www.ipez.es/RWizard/Downloads/Applications/Setup/PlotsR-manual.pdf (25-4-2018) 
Haley, K., E. Seelinger, K. Callahan Mandulak y D. J. Zajac (2010). "Evaluating the spectral distinction between sibilant fricatives through a speaker-centered approach", Journal of Phonetics, 58, pp. 548-554.

Jongman, A., R. Wayland y S. Wong (2000). "Acoustic characteristics of English fricatives", Journal of Acoustic Society of America, 108 (3), pp. 1252-1263.

Kiss, Z, (2013). "Measuring acoustic correlates of voicing in stops and fricatives": http://seas3.elte. hu/VLlxx/gkiss.html (14-06-2017).

Labov, W. (1972). Sociolinguistic Patterns. Philadelphia: University of Pennsylvania Press.

Labov, W. (1994). Principios del cambio lingüístico. Volumen II: factores internos, versión española de Pedro Martín Butragueño. Madrid: Gredos.

Lapesa Melgar, R. (1957). "Sobre el ceceo y seseo andaluces”. En Catalán Menéndez-Pidal, D. (ed.). Estructuralismo e historia. Miscelánea homenaje a André Martinet, I. La Laguna: Universidad de La Laguna, pp. 67-94.

Lasarte Cervantes, M. (2012). "Datos para la fundamentación empírica de la escisión fonemática prestigiosa de $/ \theta^{\mathrm{s}} /$ eb Andalucía". En Villena Ponsoda, J. A. y A. M. Ávila Muñoz (eds.). Estudios sobre el español de Málaga. Pronunciación, vocabulario y sintaxis.Málaga: Sarriá, pp. 129-166.

Martínez Celdrán, E. (2015). “Naturaleza fonética de la consonante 'ye’ en español”, Normas, 5, pp. 117-131.

Martínez Celdrán, E. y A. M. Fernández Planas (2007). Manual de fonética española. Articulaciones $y$ sonidos del español. Barcelona: Ariel.

Menéndez Pidal, R. (1962). "Sevilla frente a Madrid. Algunas precisiones sobre el español de América". En Catalán Menéndez-Pidal, D. (ed.). Estructuralismo e historia. Miscelánea homenaje a André Martinet, III. La Laguna: Universidad de La Laguna, pp. 99-165.

Molina García, Á. (2019). "Percepción comunitaria de la distinción fonemática de la /s/ y la / $/$ / en hablantes andaluces. Estudio en la ciudad de Málaga", Spanish in Context, en prensa.

Moya Corral, J. A. y E. J. García Wiedemann (1995). El habla de Granada y sus barrios. Granada: Universidad de Granada.

Moya Corral, J. A. Y M. Sosinski. (2015). "La inserción social del cambio. La distinción s/ $\theta$ en Granada. Análisis en tiempo aparente y en tiempo real", Lingüística Española Actual, 37/1, pp. 33-72.

Norlin, K. (1983). "Acoustic analysis of fricatives in Cairo Arabic", Working Papers, 25, pp. 113-137.

Regan, B (2017). The Effect of Dialect Contact and Social Identity on Fricative Demerger. Tesis doctoral, Universidad de Texas.

Sadowsky, S. y L. Perdomo (2018). An acoustic analysis of the ultra-high-frequency whistled /s/ of southern Chilean Spanish: https://www.linguisticsociety.org/abstract/acoustic-analysis-ultra-highfrequency-whistled-s-southern-chilean-spanish (10-02-2018).

Sawoff, A. (1980). "A sociolinguistic appraisal of the sibilant pronunciation in the city of Sevilla", Grazer Linguistische Studien, 11-12, pp. 238-262.

Svantesson, J. (1986). "Acoustic analysis of Chinese fricatives and affricates", Journal of Chinese linguistics, 14, pp. 53-70.

Tuten, D. (2003). Koineization in medieval Spanish. Berlín / Nueva York: Mouton de Gruyter.

Univaso, P., M. Martínez Soler y J. A. Gurlekian (2014). "Variabilidad intra- e inter-hablante de la fricativa sibilante /s/ en el español de Argentina", Estudios de fonética experimental, XXIII, pp. 95-125.

Vida Castro, M. A. (2013). Corpus oral complementario del español hablado en Málaga. Corpus en prensa. Universidad de Málaga. Publicación del Grupo Vernacular Urbano Malagueño.

Vida Castro, M. A. (2016). "Correlatos acústicos y factores sociales en la aspiración de /-s/ preoclusiva en la variedad de Málaga (España). Análisis de un cambio fonético en curso”, Lingua Americana, 38, pp. 15-36.

Villena Ponsoda, J. A. (2001). La continuidad del cambio lingüistico. Tendencias innovadoras y conservadoras de la fonología del español a la luz de la investigación sociolingüística urbana. Granada: Universidad de Granada. 
Villena Ponsoda, J. A., A. M. Ávila Muñoz y J. M. Sáez Sáez (1994-1995). “Modelos probabilísticos multinomiales para el estudio de ceceo, seseo y distinción de /s/ y / $\theta /$. Datos de la ciudad de Málaga”, Estudios de Lingüistica. Universidad de Alicante, pp. 391-436.

Villena Ponsoda, J. A. y M. A. Vida Castro (2012). "La influencia del prestigio social en la reversión de los cambios fonológicos. Constricciones universales sobre la variación en el español ibérico meridional. Un caso de nivelación dialectal”. En Villena Ponsoda, J. A. y A. M. Ávila Muñoz (eds.). Estudios sobre el español de Málaga. Pronunciación, vocabulario y sintaxis. Málaga: Sarriá, pp. 67-128.

Villena Ponsoda, J. A. y M. A. Vida Castro (2017). "Variación, identidad y coherencia en el español meridional. Sobre la indexicalidad de las variables convergentes del español de Málaga", Lingüistica en la Red: http://www.linred.es/monograficos_pdf/LR-monografico15-articulo1.pdf (1205-2018). 